\title{
Analysis of Instabilities in Non-Axisymmetric Hypersonic Boundary Layers over Cones
}

\author{
Fei $\mathrm{Li}^{1}$, Meelan Choudhari ${ }^{2}$, Chau-Lyan Chang ${ }^{3}$, Jeffery White ${ }^{4}$ \\ Computational AeroSciences Branch, NASA Langley Research Center, Hampton, VA 23681
}

\begin{abstract}
Hypersonic flows over circular cones constitute one of the most important generic configurations for fundamental aerodynamic and aerothermodynamic studies. In this paper, numerical computations are carried out for Mach 6 flows over a 7-degree half-angle cone with two different flow incidence angles and a compression cone with a large concave curvature. Instability wave and transition-related flow physics are investigated using a series of advanced stability methods ranging from conventional linear stability theory (LST) and a higher-fidelity linear and nonlinear parabolized stability equations (PSE), to the 2D eigenvalue analysis based on partial differential equations. Computed $\mathbf{N}$ factor distribution pertinent to various instability mechanisms over the cone surface provides initial assessments of possible transition fronts and a guide to corresponding disturbance characteristics such as frequency and azimuthal wave numbers. It is also shown that strong secondary instability that eventually leads to transition to turbulence can be simulated very efficiently using a combination of advanced stability methods described above.
\end{abstract}

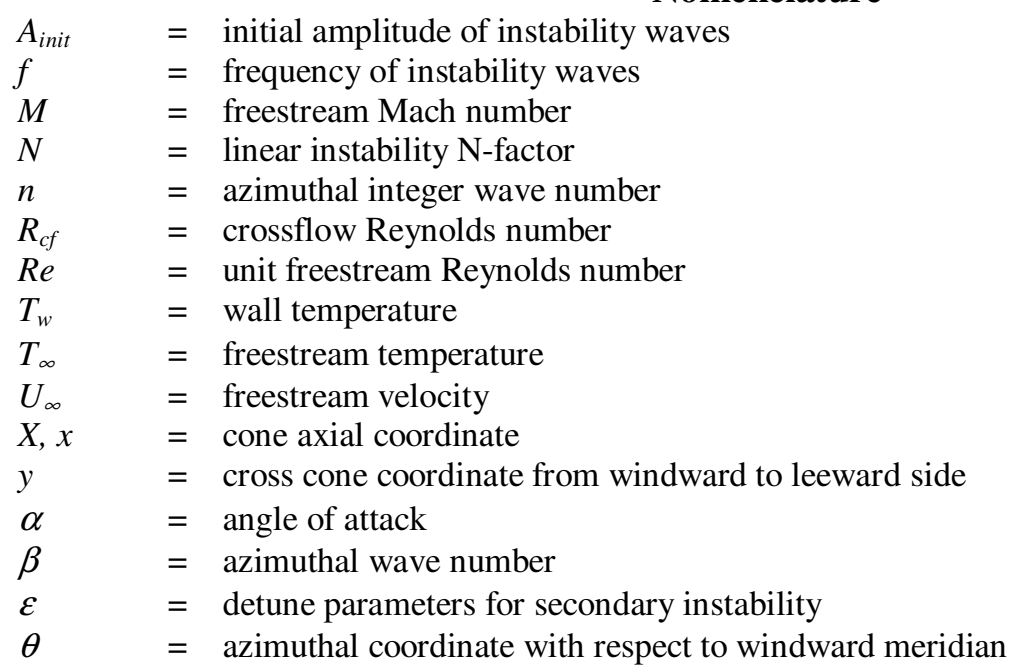

\section{Introduction}

B virtue of its influence on surface heat transfer, skin friction, and separation characteristics, boundary layer B transition from a laminar to a turbulent state tends to dominate the design and performance of hypersonic flight vehicles. Transition over realistic vehicle surfaces is often caused by surface roughness of sufficiently large amplitude. However, when the surface is relatively smooth, the laminar-turbulent transition process is initiated by linear instabilities of the laminar boundary layer. Typically, the second (or Mack) mode instability dominates transition in 2D/axisymmetric boundary layers at hypersonic speeds, although centrifugal (i.e., Görtler) instabilities may also come into play when the surface has concave curvature along the streamwise direction. Three-dimensional boundary layers involve the additional mechanisms of stationary and traveling modes of crossflow instability and, depending on the geometric configuration, the attachment line instability as well.

\footnotetext{
${ }^{1}$ Aerospace Technologist, MS 128, fei.li@nasa.gov.

Aerospace Technologist, MS 128, meelan.m.choudhari@nasa.gov, Associate Fellow, AIAA.

3 Aerospace Technologist, MS 128, chau-lyan.chang@nasa.gov, Senior Member, AIAA.

${ }^{4}$ Aerospace Technologist, MS 128, jeffery.a.white@nasa.gov, Senior Member, AIAA.
} 
Regardless of the speed regime, linear stability correlations have been quite successful in predicting the onset of transition when a single instability mechanism dominates the transition process. Mixed mode transition has, of course, been more difficult to predict. Furthermore, recent work on crossflow instability in low-speed boundary layers has exposed some of the shortcomings of applying purely linear predictive models to transition in 3D boundary layers by revealing the importance of nonlinear effects during crossflow dominated transition. ${ }^{1}$ The canonical configuration of a circular cone at angle of attack (AOA) includes the necessary elements to study both mixed mode transition and crossflow development in the context of a high-speed boundary-layer flow. ${ }^{2-9}$ Instability mechanisms for a supersonic or hypersonic boundary layer flow over an elliptic cone have been investigated in Refs. 10-20. While the elliptic cone configuration supports similar instability mechanisms as the circular cone at angle of incidence, the details of transition patterns can be rather different as noted in Ref. 20.

The availability of quiet hypersonic facilities opens up the possibility of a detailed study of the abovementioned instability phenomena in a controlled environment. ${ }^{21,22}$ Such experiments must, however, be guided by a detailed analysis of the flow field and its stability characteristics. The objective of this ongoing effort is to provide that characterization in support of the experiments at Purdue University, ${ }^{7,9,23}$ to pave the way for jointly addressing a few of the outstanding issues related to transition mechanisms in hypersonic boundary layers over nominally smooth surfaces. To that end, both linear and nonlinear evolution of a variety of instability mechanisms relevant to hypersonic vehicles have been examined in the context of canonical flow configurations.

A brief summary of the flow configurations of interest is given in Section II, which also describes the analysis codes used in this study. Stability characteristics of the circular cone boundary layer for two different angles of attack ( $\alpha=3$ and 6 degrees, respectively) are examined in Section III. Section IV is devoted to the Purdue compression cone configuration, ${ }^{23-25}$ which involves an additional instability mechanism in the form of Görtler vortex instability. The original aim was to examine the effect of a nonzero angle of incidence and, hence, a nonzero crossflow on the Görtler modes. However, it was found that this particular model is not well suited for transition studies at angles of attack equal to 1 degree and above, because of the small cone angle near the leading edge, the thick leeward boundary layer cannot negotiate the adverse pressure gradient along the axial direction and separates. The flow characteristics and, in particular, the Görtler instability amplification along the windward and leeward lines is addressed for an angle of incidence equal to 0.5 degrees. Most of the effort is then devoted to extending the previous analysis ${ }^{24}$ of nonlinear transition scenarios for the axisymmetric compression cone. A brief summary of the results is provided in Section V.

\section{Flow Configuration and Analysis Codes}

\section{A. Circular Cone at Angle of Incidence}

The first flow configuration of interest corresponds to a circular cone at angle of attack to a hypersonic stream. The primary configuration for the Purdue experiments corresponds to a 0.457 meter long ( 18 inches), nominally sharp nosed cone (modeled with a nose radius of $10^{-4}$ times the cone length) with a half angle of 7 degrees and an angle of attack equal to 3 or 6 degrees. The flow conditions correspond to a free-stream Mach number of $M=6$, unit Reynolds number $\mathrm{Re}=10.8 \times 10^{6}$ per meter, and free-stream temperature $\mathrm{T}_{\infty}=52.44 \mathrm{~K}$. The temperature of the model surface corresponds to $\mathrm{T}_{\mathrm{w}}=300 \mathrm{~K}$.

\section{B. Compression Cone}

The second flow configuration studied herein corresponds to the Purdue Mach 6 compression cone ${ }^{19}$ with a stagnation pressure of $930.1 \mathrm{kPa}$, a stagnation temperature of $766.8 \mathrm{~K}$, and unit Reynolds number of $10.8 \times 10^{6}$ per meter. The cone is 0.47 meters long and has a constant surface radius of curvature equal to 3 meters in the axial direction. It tangentially joins a small nose sphere of radius $0.16 \mathrm{~mm}$ at an initial half-angle of 1.4 degrees. The boundary layer over this surface is susceptible to moderate Görtler and first mode instabilities and a strong second mode instability. For this compression cone configuration, a previous set of computations ${ }^{24}$ focused on the transition route via interactions between (i) the axisymmetric second mode and the Görtler mode and (ii) a pair of oblique second mode instabilities which is not as highly amplified as the axisymmetric second mode but has linear $\mathrm{N}$-factor values in excess of 9 . In this paper, we continue this study by exploring other potential scenarios for the onset of transition in the compression cone boundary layer. Of particular interest are the secondary instabilities of the axisymmetric second mode; however, the potential for oblique mode breakdown of first mode waves and highfrequency secondary instabilities of Görtler vortices are also briefly examined. 
The steady flow over the cones is computed using the VULCAN ${ }^{26}$ (Viscous Upwind aLgorithm for Complex flow ANalysis) software package. The code solves the unsteady, conservation equations appropriate for laminar or turbulent flow of calorically or thermally perfect gases with a spatially second order accurate cell-centered finite volume scheme. Efficient utilization of parallel architectures is realized through calls to MPI (Message Passing Interface) routines using a SPMD (Single Program Multiple Data) paradigm. A variety of upwind schemes are available for fine-tuning of numerical dissipation and shock capturing in order to ensure solution accuracy for stability computations. The inviscid fluxes were constructed using the MUSCL $K=0$ scheme, the van Albada gradient limiter $^{27}$ and the Low Dissipation Flux Split Scheme (LDFSS) of Edwards. ${ }^{28}$ The cell face gradients required to construct the viscous fluxes were obtained using an auxiliary control volume approach that results in a compact viscous stencil that produces a second order accurate approximation of the full Navier-Stokes viscous fluxes. The solutions were relaxed in pseudo time to steady state using the 3-D ILU(0) scheme ${ }^{29}$ with a constant CFL number on the order of 50 . Grids of $345 \times 257 \times 353$ and $345 \times 129 \times 353$ in the axial, azimuthal, and wall-normal directions, respectively, were used for the straight circular cone at angle of attack, whereas a grid of $2241 \times 385$ points in the streamwise and wall-normal directions, respectively, was used for the compression cone configuration at zero angle of attack under the axisymmetric assumption. Mean flow computations for the similar elliptic cone configuration were cross-validated against LAURA ${ }^{30}$ solutions for various grid sizes during the work in Ref. 20. Thus, no extensive grid convergence analysis was performed for the mean flows used herein.

Linear and nonlinear development of the instability modes of interest are computed using conventional linear stability theory (LST) or the parabolized stability equations (PSE) method as implemented in the Langley Stability and Transition Analysis Code(s) (LASTRAC). ${ }^{31}$ The LASTRAC software suite provides a set of transition prediction tools that are particularly suitable for the studies of a variety of instability mechanisms present in a hypersonic boundary layer over a circular cone. Linear growth characteristics of secondary instability modes are predicted using the methodology outlined in Ref. 32. Sutherland's law is assumed to model the viscosity variation for both the base flow and the instability waves. Stokes law is assumed for bulk viscosity. Extensive experience with these codes has established the grid resolution required to obtain nearly grid-independent solutions. Hence, no grid convergence analysis was deemed necessary for the results obtained using these codes. Of course, because of the Fourier discretization in the azimuthal coordinate and time, grid convergence in those directions was ensured by requiring a sufficient decay (typically, three orders of magnitude or more) in Fourier amplitudes from the highest amplitude Fourier modes to the tail of the spectrum.

\section{Circular Cone at Angle of Incidence}

Stability and transition characteristics are investigated for the Purdue circular cone configuration as described in the previous section. Two flow incidence angles, 3 degrees and 6 degrees, are studied computationally to assess the corresponding effects. Figure 1 shows a schematic of the base flow at 6 degree angle of attack. Limiting streamlines (also referred to as skin friction lines) plotted in Fig. 1a reveal the azimuthal movement of the boundary layer flow towards the leeward meridian, which leads to a substantial thickening of the boundary layer near the leeward plane of symmetry as depicted by the blue colored bulge in the axial momentum contours at the downstream end of the cone. The inclination of the limiting streamlines (Fig. 1a) relative to the inviscid streamlines near the edge of the boundary layer (Fig. 1b) indicates the presence of strong crossflow inside the boundary layer. The axial momentum contours along the symmetry plane (Fig. 1a) indicate the shock front within the windward plane and the substantially weaker shock within the leeward plane, where the surface deflection relative to the oncoming stream is reduced to just 1 degree. Consistent with this shock pattern, the edge Mach number is relatively lower near the windward meridian, opening the possibility of first mode instability in that region.

Distribution of the crossflow Reynolds number, $R_{\mathrm{cf}}$, over the cone surface is shown in Fig. 2. The maximum crossflow velocities occur near $\theta=135$ degrees; however, the maximum values of the crossflow Reynolds number are observed closer to the leeward plane. This particular trend is attributed to the thickening of the boundary layer as the symmetry plane is approached. The relatively large values of the crossflow Reynolds number are consistent with the strong crossflow instability that might be expected at a 6 degree angle of attack. Maximum crossflow Reynolds numbers are roughly one half of the values shown in Fig. 2 for the 3 degree angle of attack case. Weaker crossflow instability for the latter case is expected.

\section{A. Streamwise Instability at the Symmetry Planes}


Mean flow along both windward and leeward symmetry planes appears to be similar to a supersonic 2D boundary layer. However, as discussed previously, the thickening of the boundary layer near the leeward plane of symmetry results in a distinct mushroom-like streak structure as shown in Fig. 3. Strong shear layers near the edge of the boundary layers can substantially change the stability characteristics. For such two-dimensional structure on the cross-stream plane, the 2D eigenvalue approach, as used in the following section, is more appropriate. Nevertheless, conventional linear stability and PSE analyses were carried out to gain understanding of the flow physics and stability characteristics on the symmetry plane. The post-shock Mach number varies slightly along the streamwise direction. For the 6 degree angle of attack case, the boundary-layer edge Mach number is approximately 4.7 and 5.8 along the windward and leeward symmetry plane, respectively. Due to a weaker leading edge shock, boundary-layer edge Mach numbers are 5.13 and 5.68 for the 3 degree angle of attack case. Both first- and secondmode instability waves are present at these Mach numbers.

Several methods are normally used for transition correlation of first-mode instability waves. For a conical configuration, $\mathrm{N}$-factor envelops for instability waves with various frequency and constant azimuthal wave numbers appear to be physically more appropriate. Figure 4 shows the resulting disturbance growth rates at various frequencies for both first- and second-mode disturbances. The second-mode waves apparently have a much higher overall growth rate. However, oblique first modes with a constant azimuthal wave number have sustained growth over a longer streamwise distance. As a result, the overall $\mathrm{N}$-factor of the first mode, shown in Fig. 5, is only slightly smaller than that of the second mode. Corresponding disturbance frequencies and azimuthal wave numbers are also shown in the same figure. The disturbance frequency range for the first mode is approximately $40-120 \mathrm{kHz}$, much lower than that shown in Fig. 5b. The maximum $\mathrm{N}$-factor value near the end of the model is only about 4 and 4.8 for first- and second-mode waves, respectively. It is thus questionable whether the flow would transition in the windward symmetry plane under a quiet condition. $\mathrm{N}$-factor envelope curves for the 3-degree angle of attack case are shown in Fig. 6 for comparison. Qualitatively, they resemble those for the higher angle of attack. However, the second mode waves appear to be more unstable, with a maximum $\mathrm{N}$-factor value of about 6.2 , as expected due a high boundary layer edge Mach number for the 3 degree angle of attack case.

Boundary-layer edge Mach numbers at the leeward side symmetry plane are approximately 5.7-5.8. At this Mach number, the dominant mechanism is expected to be of the second-mode type. Due to the distinct mushroomlike bulge structure at this symmetry plane, some kind of shear-layer instability is also expected downstream. Figure 7 shows the computed second-mode $\mathrm{N}$-factor envelopes for both cases. Both $2 \mathrm{D}$ and slightly oblique modes with an azimuthal wave number of less than 10 are found to be unstable and give rise to significant disturbance growth. For the larger angle of attack, non-parallel effects are more significant due to the thicker boundary layer. It is interesting to note that non-parallelism has opposite effects for the larger and smaller angle of attack. In the case of 6 degree angle of attack the PSE result, which include non-parallel effects, predicts smaller $\mathrm{N}$-factors than the quasi-parallel result. On the other hand, in the 3 degree angle of attack case, the opposite is true. However, the dominant twodimensional structure in the leeward symmetry plane implies that a local eigenvalue analysis which ignores the spanwise variation could not account for all the flow physics. A 2D eigenvalue analysis is more appropriate and will be studied in a follow on work. The difference in boundary layer thickness also results in a much lower unstable frequency range (around 50-250 kHz) for the 6-degree angle of attack case. In contrast, the maximum Nfactor values are much larger along the leeward plane of symmetry. They reach about 16 and 12 for 6 degree and 3 degree angle of attack, respectively. These relatively large values suggest that transition is more likely to take place even in a quiet environment.

Given a disturbance amplitude distribution either from receptivity analyses or experimental measurements, transition locations can be computed by using the nonlinear PSE. As a preliminary assessment of the nonlinear effect, an unstable second-mode disturbance with a frequency of $100 \mathrm{kHz}$ was arbitrarily assigned different initial amplitudes. The resulting streamwise velocity perturbation evolution is plotted in Fig. 8 for initial amplitudes (measured in peak temperature perturbation) of $1 \%$ and $0.1 \%$ of the freestream temperature. In both cases, the 2D fundamental mode of frequency $100 \mathrm{kHz}$ saturates and then decays. The strong shear layer causes the decaying primary mode to grow again further downstream. Peak time-RMS amplitudes reach about $2.5 \%$ and $1 \%$ for the primary mode and about $4 \%$ and $1.5 \%$ for the mean flow distortion mode. At such amplitudes, secondary instability, as will be discussed later, can lead to strong nonlinear interaction and breakdown to turbulence. Although not yet investigated systematically, oblique second mode breakdown is also a candidate for transition mechanism, given the fact that a slightly oblique disturbance with an azimuthal wave number of approximately 10 still has significant growth. 


\section{B. Crossflow Instability over the Cone Surface}

Results pertaining to the amplification of the stationary crossflow instability along the cone surface are shown in Fig. 9. The N-factor contours plotted in Fig. 9a correspond to an envelope of the integrated amplification ratios for stationary vortex instabilities of several selected azimuthal wave numbers. Figure $9 \mathrm{~b}$ shows the data from in Fig. $9 \mathrm{a}$ when plotted in the $\mathrm{x}-\theta$ plane. As expected, the crossflow instability is rather strong, with $\mathrm{N}$-factor values approaching 20 for the stationary modes. The most unstable stationary modes correspond to azimuthal wavenumbers in the range of $\beta=45$ to 55. This is in good agreement with the experimentally measured azimuthal wave number of approximately 45 at an axial coordinate of $0.38 \mathrm{~m}$ using temperature sensitive paint (see Ref. 9). The peak crossflow growth, as predicted on the basis of growth rate integration along (approximate) inviscid streamlines, is observed to occur in the neighborhood of $\theta=130-140$ degrees. This angle is larger than the value found earlier for a Mach 3.5 cone at 2 degrees angle of attack. ${ }^{2}$ The shift towards a larger angle for the present configuration is believed to be a combined effect of the larger Mach number and angle of attack. The N-factor distribution over the cone surface computed for stationary crossflow instability provides an assessment of possible transition fronts on the surface with a pre-determined $\mathrm{N}$-factor value. For unknown reasons, the computed transition fronts with a prescribed N-factor shown in Fig. 9 do not appear to agree with the experimental measurements in Ref. 9 using temperature sensitive paint under noisy wind tunnel conditions. The lower-branch neutral locations where stationary crossflow just becomes unstable can also provide guidance for excitation of instability waves. Since any disturbance trigged by roughness elements placed too far upstream of the neutral location will decay exponentially, it would be appropriate, as a first estimate to place artificial roughness elements in its vicinity in order to excite the stationary modes in a controlled fashion. Indeed, experiments of this type would help address several unanswered issues related to disturbance amplification in fully 3D boundary layers and, hence, help refine some of the ad hoc assumptions in transition prediction based on classical stability theory. ${ }^{9,10}$ It is important to mention that the crossflow instability depends on the characteristics of sources that trigger it, comparisons with instability growth excited by specific types of roughness elements are ongoing.

To assess non-parallel effects, linear PSE calculations were performed along one of the integration paths shown in Fig. 10a. The stationary crossflow instability mode with an azimuthal wave number $\beta=42$ was computed by quasi-parallel linear stability theory with all curvature terms (both streamwise and transverse curvatures) included (QPWC - Quasi-Parallel With Curvature) and the linear PSE. The resulting N-factors depicted in Fig. 10b show very minimal difference. This weak non-parallel effect is similar to that observed in the HiFire5 NASA Langley wind tunnel model. ${ }^{19}$

Traveling crossflow instability was computed along streamline integration paths shown in Fig. 10a. The disturbance growth rate is maximized with respect to the spanwise wave numbers at each location. This $\mathrm{N}$-factor integration is similar to the conventional saddle point method, except that the integration paths are along the inviscid streamlines rather than the group velocity directions. Figure 11 shows the computed dominant traveling crossflow instability waves at three different downstream locations. It clearly shows that dominant frequency in terms of peak local growth rate changes from $25 \mathrm{kHz}$ upstream to near $10 \mathrm{kHz}$ downstream. The corresponding optimized azimuthal wavenumber is relatively unchanged along the trajectory and remains approximately 40. Figures 12a and $12 \mathrm{~b}$ depict the computed $\mathrm{N}$-factor distribution on the surface along with the corresponding optimized frequency and azimuthal wave numbers, respectively. The traveling crossflow $\mathrm{N}$ factors are in general larger than those for stationary crossflow. The frequency range for the traveling crossflow instability with an $\mathrm{N}$-factor value between 10 and 20 is found to be from $25 \mathrm{kHz}$ to $60 \mathrm{kHz}$ approximately. At $\mathrm{N}=10$, the frequency is around $40 \mathrm{kHz}$ and an azimuthal wave number of approximately 28. At a larger $\mathrm{N}$-factor value of 16 to 20 , the azimuthal wave number is increased to approximately 35 to 45 .

The computed stationary crossflow instability waves for the 3 degree angle of attack case are shown in Fig. 13. Qualitatively, it appears to be similar to the 6 degree angle of attack case shown in Fig. 9a. Maximum N-factor values are substantially smaller at the smaller angle of attack. The corresponding azimuthal wave number varies from 33 to 48 along the ridge of the contours between $\mathrm{N}$-factor values of 6 and 9 . The traveling crossflow instability results are also shown in Fig. 14. Maximum N values only reach about 18 in this case. Similar N-factor contours plotted in the $x-\theta$ plane shown in Fig. 14b show that maximum growth takes place at around 125 degrees, slightly smaller than for the 6 degree case. The optimal growth disturbance frequency is around $50-60 \mathrm{kHz}$ in the vicinity of the apex of the $\mathrm{N}=10$ contour. The corresponding azimuthal wave number is approximately 40-50. Both frequency and azimuthal wave numbers are only slightly higher than that for the 6 degree case. The relatively 
smaller $\mathrm{N}$-factor values for the 3 degree angle of attack case suggest that transition may or may not be observed experimentally if performed in a quiet supersonic wind tunnel.

\section{Secondary Instability of Second Mode on Compression Cone}

For hypersonic boundary layers, second mode instability can be a significant factor leading to transition. Due to the concave longitudinal curvature of the compression cone described in Section II, the boundary layer over this cone also supports the Görtler instability in addition to the first, second and crossflow mode instabilities over the straight circular cone from Section III. Consequently, the onset of laminar-turbulent transition over the compression cone may occur via either one of several different routes, such as (1) Görtler-second mode interactions, (2) oblique second mode interactions, (3) secondary instability of second mode, (4) oblique breakdown of first mode instabilities, (5) secondary instability of the Görtler vortex, and (6) interactions between axisymmetric second mode and oblique first modes. Routes (1) and (2) of these were addressed in Ref. 24. A majority of this section is devoted to route (3), i.e., the secondary instability of axisymmetric, finite amplitude, second mode instability. However, the secondary instability of Görtler vortices and oblique first mode interactions are also discussed briefly.

The secondary instability of a primary instability is a well-defined and well-known phenomenon. However, in the context of secondary instability of the second mode perturbations, it is important to distinguish between second mode and secondary instability, so that no confusion will result because of the word "second". The second mode is an instability mode of the laminar boundary layer, while the secondary instability of second mode is an instability mode to which the second mode becomes susceptible when its amplitude exceeds a certain threshold. For secondary instability to exist, the second mode must reach a sufficiently large amplitude and, in this context, the second mode may be referred to as the primary instability.

\section{A. Nonlinear Evolution of Axisymmetric Second Mode Waves}

Figure 15a shows the linear $\mathrm{N}$-factors for axisymmetric second modes of various frequencies with zero azimuthal wavenumber predicted by linear PSE. It is seen that modes in the neighborhood of $300 \mathrm{kHz}$ frequency are most amplified. It is well known that a $2 \mathrm{D}$ or axisymmetric second mode is more unstable than an oblique second mode of the same frequency. This is confirmed in Fig. 15b, where N-factors of second modes of $300 \mathrm{kHz}$ with different azimuthal wavelengths predicted by linear PSE are plotted and it can be seen that the second mode with zero azimuthal wavenumber has the largest $\mathrm{N}$-factor. All $\mathrm{N}$-factor curves in Fig. 15 are monotonically increasing, and a maximum $\mathrm{N}$-factor of 19 is reached at the end of the cone, indicating very strong second mode growth.

Finite amplitude growth of second mode perturbations is investigated next. Four different initial amplitudes of the second mode with a frequency of $300 \mathrm{kHz}$ are used to start nonlinear PSE computations. Fig. 16 displays the streamwise evolution of modal amplitude of the fundamental harmonic in each of these cases, along with the linear PSE prediction. The amplitudes in Fig. 16 are normalized by their respective initial values. Thus, all curves initially coincide because the effects of disturbance nonlinearity have not set in at these small amplitudes and therefore the linear theory is valid to leading order. It is well known that nonlinear effects will come into play as the disturbance amplitude increases, and the second mode evolution will begin to depart from the linear theory prediction. The second mode disturbance eventually saturates, i.e. settles down and grows or decays algebraically instead of exponentially. However, the particular case under discussion shows unusual behavior that has not been seen before. Whereas nonlinear saturation would be expected when the fundamental amplitude becomes large, the disturbance amplitude plummets, with the rates of decay comparable to their respective initial, exponential growth rates. Effectively, increased nonlinearity progressively brings the upper neutral branch to a forward location. The meanflow correction term (not shown), however, does not decay as rapidly and remains at relatively large amplitudes up to the end of the cone. Perhaps, it is the change in the mean flow that causes the rapid decay of the finite amplitude second mode instability. Further investigation of this phenomenon is underway.

\section{B. Validation of Growth Rate Predictions for Secondary Instability}

In spite of the rapid premature decay of the second mode, there is still a streamwise region in which the second mode amplitude is large enough to sustain secondary instability. Secondary instability of second mode instabilities was first studied by $\mathrm{Ng}$ and Erlebacher ${ }^{33}$ for a supersonic boundary layer at Mach 4.5. Since the second mode instability grows spatially in the streamwise direction, it is assumed that the change in amplitude over one second mode wavelength is negligible and the flow field can be considered to be locally periodic. The secondary instability is then governed by a set of partial differential equations with periodic coefficients in the streamwise direction. As a result, an eigenfunction of the set of PDEs takes on a certain periodic form. ${ }^{34}$ Two special cases of the secondary 
instability are the fundamental and subharmonic modes with eigensolutions that have, respectively, the same wavelength and twice the wavelength of the second mode base flow. A more general solution can have a wavelength that is not necessarily an integral multiple of the second mode base flow wavelength; this is termed the detuned mode. A parameter $\varepsilon$ can be defined to characterize the periodic properties of the secondary instability, with $\varepsilon=0$ and 1 indicating the fundamental and the subharmonic modes, respectively. For $0<\varepsilon<1$, the secondary instability is detuned. ${ }^{34}$

The base flow in the earlier analysis of secondary instabilities for a Mach 4.5 boundary layer was composed simply of a second mode linear eigenfunction with a small but finite amplitude superimposed on the laminar boundary layer flow as an approximation to the distorted boundary-layer flow field. As a validation of the secondary instability analysis tools used in the present work (the 2D eigenvalue solver), computations on the same flat-plate flow configuration of $\mathrm{Ng}$ and Erlebacher ${ }^{33}$ is carried out, and the agreement between their results and the results computed by modifying the planar, partial differential equations based eigenvalue solver from Ref. 32 are very good (Fig. 17a). Additional comparisons of a similar nature were obtained for secondary instability of first modes waves in the same Mach 4.5 boundary layer (Fig. 17b), as well as for the fundamental mode of secondary instability in a Mach 1.6 boundary layer (not shown). In the secondary instability computations for the compression cone configuration in this paper, the base flow for secondary instability is obtained by solving nonlinear PSE, accounting for all dominant harmonics of the second mode instability, including distortion, if any, of the primary (i.e., fundamental) harmonic.

\section{Linear Predictions for Subharmonic and Fundamental Modes of Secondary Instability}

To study the secondary instability, the evolution of a second mode of frequency $300 \mathrm{kHz}$ is first computed by nonlinear PSE with a small initial amplitude $\left(10^{-5}\right)$ in streamwise velocity (normalized by the freestream velocity). Then nonlinear PSE results are used as the base flow for secondary instability. Figure 18 shows the growth rates at different azimuthal wavenumbers of three different modes of secondary instability, namely, a fundamental, a subharmonic and a detuned $(\varepsilon=0.5)$ mode, at a streamwise location of $x=0.35 \mathrm{~m}$, which is near the peak of the second mode amplitude (see the red curve in Fig. 16). The fundamental mode has the highest growth rates, while the subharmonic and the detuned modes have comparable growth rates. The unstable spectra span a wide range of azimuthal wavenumbers $(n)$ with peak growth at approximately $n=60$ for the fundamental mode. All three types of secondary instability are approximately phase locked with the second mode primary instability.

Figure 19 shows the $\mathrm{N}$-factors of fundamental and subharmonic modes of secondary instability at various azimuthal wavenumbers. These curves were obtained by integrating the local amplification rates computed by the 2D eigenvalue solver, starting from the onset of secondary growth for each mode of interest. For both types of secondary instabilities, the $n=60$ modes reach the highest $\mathrm{N}$-factors, with the maximum $\mathrm{N}$-factor for the fundamental mode at 13.5 and that for the subharmonic mode at 11, at the end of the cone, indicating strong secondary instability growth. Modes with neighboring azimuthal wavenumbers (i.e. $n=80$ ) also achieve very high amplification factors, very close to those of the $n=60$ modes. Figure 20 shows the shapes of the fundamental and subharmonic eigenfunctions in terms of colored contours of the streamwise velocity fluctuation, while the background white lines are contours of the mean streamwise shear. The nearly horizontal band of background white lines in Fig. 20 indicate the concentration of strong shear created by a finite amplitude second mode, which coincides with the maximum amplitudes of secondary instability.

\section{Nonlinear Evolution of Subharmonic Mode of Secondary Instability}

The linear 2D eigenvalue solver used here for secondary instability does not account for non-parallelism and surface curvature effects. On the other hand a PSE computation includes both of these effects. Furthermore, nonlinear PSE computations can trace the nonlinear evolution of secondary instability up to the onset of transition. To this end, nonlinear PSE computations were initiated for a second mode of frequency $300 \mathrm{kHz}$ and an initial streanwise velocity amplitude of $10^{-4}$ (normalized with free-stream velocity) at approximately $x=0.095 \mathrm{~m}$, and a subharmonic secondary instability mode of $n=80$ computed by the 2D eigenvalue solver at $x=0.26 \mathrm{~m}$ is inserted when the nonlinear PSE marching reaches this location, slightly downstream of the neutral point of the secondary instability. Figure 21a shows the nonlinear evolution of modal amplitudes of the second mode (red lines) and the secondary instability (green lines) downstream of $x=0.26$. Also plotted in Fig. 21a is the secondary instability amplitude predicted by linear theory, which is represented by solid black dots. Initially the linear theory agrees well with the nonlinear PSE results, despite its lack of non-parallelism and curvature effects. Eventually, however, larger differences appear between the secondary instability theory and the PSE computations, obviously due to nonlinear 
effects. Similarly, Figure 21b shows a comparison between the streamwise evolution of peak RMS amplitude of the secondary instability at a given station and the corresponding prediction from linear secondary instability theory.

The onset of transition is often characterized by a sharp rise in the mean wall shear. The evolution of mean wall shear in the presence of secondary instability of various initial amplitudes is shown in Fig. 22. The wall-shear curve with only the second mode present (no secondary instability) is shown in black and serves as a reference for determining the onset of transition. For the secondary instability with the smallest initial amplitude (the red curve), the wall-shear follows the reference curve for most of its history, but rises sharply at approximately $x=0.44 \mathrm{~m}$, just before the end of the cone is reached. The curve corresponding to larger initial amplitude begins to deviate slightly from the reference curve before rising sharply at approximately $x=0.40 \mathrm{~m}$.

Figure 23 shows the RMS contours of streamwise velocity fluctuations of secondary instability at three selected cross-sections on the cone. Initially the RMS contours consist of large islands near the edge of the boundary layer. As the onset of transition near $x=0.44 \mathrm{~m}$ is approached, these islands begin to break up into smaller scales.

The results in this section indicate the subharmonic secondary instability is a viable route to transition in the compression cone boundary layer. Since the fundamental mode of secondary instability appears to have a higher linear growth potential than the subharmonic modes, it will be important to determine their role in transition. Such analysis is ongoing and the results will be reported in a future paper.

The subharmonic secondary instability does not involve a strong, stationary vortex component and, therefore, may not account for the stationary vortex signature seen from the experimental visualization of the surface flow via temperature sensitive paint (TSP). ${ }^{23}$ The fundamental mode of secondary instability, on the other hand, does involve an $\mathrm{O}(1)$ stationary vortex component as part of its mode shape and, hence could possibly account for the abovementioned stationary vortices seen in the TSP images. ${ }^{23}$

Figure 24 shows the linear $\mathrm{N}$-factors of Görtler instability at various azimuthal wavenumbers. A maximum Nfactor of approximately 5.5 is reached at the end of the cone by a Görtler mode of azimuthal wavenumber $n=50$. Since this not a large N-factor value, transition via the breakdown of Görtler vortices will require relatively large initial Görtler amplitudes. For secondary instability analysis, a Görtler vortex of $n=48$ and initial amplitude 0.01 of streamwise disturbance velocity is traced by nonlinear PSE computations, starting at $x=0.95 \mathrm{~m}$ downstream of the cone tip. The amplitude of the Görtler vortex reaches approximately $24 \%$ of the freestream velocity at $x=0.43 \mathrm{~m}$, where strong secondary instability exists. Upstream of this location, the neutral point for the secondary instability is at approximately $\mathrm{x}=0.39 \mathrm{~m}$. Figure 25 shows the spectra secondary instability modes of Görtler vortices, which covers a frequency range from close to 0 to approximately $400 \mathrm{kHz}$. Three secondary instability modes are shown with the most amplified mode being symmetric (even) about the plane of symmetry between a counter-rotating Görtler vortex pair and the two less unstable modes are both anti-symmetric (odd).

The effect of a small angle of attack ( 0.5 degrees) on the linear amplification of Görtler modes is considered next. Skin friction lines along the cone surface in Fig. 26 indicate the presence of a mild crossflow in this case. Cross-plane contours of streamwise velocity at $\mathrm{x}=0.2 \mathrm{~m}$ are shown in Fig. 27. A comparison of the linear $\mathrm{N}$-factor curves in Figs. 28a and 28b shows the effect of a small angle of attack on N-factor of linear Görtler instability modes. The leeward side is slightly more unstable. A likely cause for the stronger Görtler instability along the leeward line corresponds to the thickening of the boundary layer, resulting in a higher Görtler number.

Finally, it can be shown that, for the compression cone at an angle of attack of 0 degrees, oblique first mode interactions are an unlikely transition scenario. Figure 29 shows the wall shear stress in the case of oblique first mode interactions between a pair of oblique $80 \mathrm{kHz}$ first mode waves of the same azimuthal wavenumber, $n=12$, but of opposite orientation. It is seen that very large amplitudes are necessary to initiate transition indicated by the rise in wall shear.

\section{Summary and Concluding Remarks}

Transition studies have been performed for a Mach 6 flow over a circular cone at 3 degree and 6 degree incidence angles. The laminar steady-state mean flows were obtained by the VULCAN Navier-Stokes equations solver. Numerical parameters and grid spacing were chosen to ensure good resolution of boundary-layer profiles as well as in the inviscid region containing the nose shock. A variety of instability wave mechanisms show up in both 
configurations. In addition to the presence of both stationary and traveling crossflow vortex instabilities over a large portion of the cone surface, streamwise instabilities including first- and second-mode waves become unstable along both the windward and leeward planes of symmetry. Three-dimensional effects on the cross-stream planes result in a significantly thickened boundary layer and a mushroom-like structure near the leeward symmetry which further introduces shear-layer instability around the edges of the boundary layer.

Linear analyses using both LST and PSE were carried out to identify characteristics of all instability mechanisms above. It was found that the maximum stationary crossflow $\mathrm{N}$-factors reach about 12 and 20 for the 3 degree and 6 degree cases, respectively. Traveling crossflow waves computed by maximized growth rates achieve a peak Nfactor value of about 18 and 24, respectively. Both first- and second-mode disturbances were found to be unstable along the windward symmetry plane. For both incident angles, the maximum $\mathrm{N}$-factor only reaches about 4 and 6 for first- and second-mode disturbances, respectively. Such small N-factor values suggest that transition is unlikely to take place in the windward symmetry plane under a quiet environment and competition between these two instability modes would determine which mode dominates when the environment is noisy. In contrast, the leeward symmetry plane is dominated by the second-mode disturbances due to a higher boundary-layer edge Mach number of around 5.8, with the maximum $\mathrm{N}$ factor reaching about 13 and 17 for two flow incident angles, respectively. Strong growth of the 2D and oblique second-mode disturbances could lead to amplitudes that are large enough to trigger either oblique-mode breakdown or rapid secondary instability growth, as identified in the compression cone studies shown in Section IV. Based on the above results, the major effect of a larger flow incident angle is primarily on the crossflow instability and it has relatively minor effect on the streamwise instability mechanisms on the planes of symmetry.

The analysis of transition scenarios over the Purdue, Mach 6 axisymmetric compression cone configuration was extended to include secondary instabilities of the dominant, axisymmetric second mode wave, the high-frequency instabilities of Görtler modes, and the nonlinear interactions between oblique first mode waves. Results obtained from secondary instability theory using a 2D eigenvalue approach showed that both subharmonic and fundamental modes of secondary instability give rise to secondary mode $\mathrm{N}$-factors of greater than 8 , even with a relatively small primary second mode streamwise velocity amplitude of $1 \times 10^{-5}$ relative to the freestream velocity. Thus, either of these secondary instability modes can potentially lead to transition, provided that they are excited to an adequate initial amplitude from the disturbance environment. The analysis also showed that the predicted azimuthal wavenumbers of these secondary instabilities with the highest linear amplification rates are only slightly higher than that of the linearly most unstable Görtler modes. The overlap in these wavenumbers increases the likelihood of excitation of secondary instability in a natural or perturbed environment. Nonlinear PSE computations were performed to establish the transition onset locations for the assumed set of initial disturbance amplitudes. Previous computations in Ref. 25 have shown that the initial amplitudes of the primary second mode waves can be predicted, in principle, if sufficient knowledge of the background disturbances is available. Combined with the analysis presented herein, it appears that high-fidelity predictions of transition for hypersonic boundary layers via the secondary instability route are within sight, analogous to that of crossflow transition in swept wing boundary layers at lower Mach numbers. ${ }^{35}$

\section{Acknowledgments}

The authors gratefully acknowledge technical communications with Drs. F. Greene, A. Norris, and T. Jentink at NASA LaRC, Prof. S.P. Schneider of Purdue University, and Dr. E. Swanson from Northrop Grumman Corporation.

\section{References}

1. Saric, W.S., Reed, H.L., and White, E.B., "Stability and Transition of Three-Dimensional Boundary Layers," Ann. Rev. Fluid Mech., Vol. 35, pp. 413-40, 2003.

2. King, R. A., "Three-Dimensional Boundary-Layer Transition on a Cone at Mach 3.5," Experiments in Fluids, Vol. 13, No. 5, pp 305-314, 1992.

3. Stetson, K.F., Thompson, E.R., Donaldson, J.C., and Siler, L.G., "Laminar Boundary Layer Stability Experiments on a Cone at Mach 8 - part 3: Sharp Cone at Angle of Attack," AIAA Paper 85-0492, 1985.

4. Malik, M. R. and Balakumar, P., "Instability and Transition in Three-Dimensional Supersonic Boundary Layers," AIAA Paper 1992-5049, 1992.

5. Hanifi, A. and Dahlkild, A.A., "Some Stability Characteristics of the Boundary Layer on a Yawed Cone," AIAA Paper 1993-3048, 1993. 
6. Schneider, S.P., "Hypersonic Laminar-Turbulent Transition on Circular Cones and Scramjet Forebodies," Prog. in Aerospace Sc., Vol. 40, pp. 1-5, 2004.

7. Swanson, E., "Boundary-Layer Transition on Cones at Angle of Attack in a Mach 6 Quiet Tunnel," Ph.D. Dissertation, School of Aeronautics and Astronautics, Purdue University, 2008.

8. Balakumar, P., "Stability of Supersonic Boundary Layers on a Cone at an Angle of Attack," AIAA Paper 20093555, 2009.

9. Swanson, E. and Schneider, S.P., "Boundary-Layer Transition on Cones at Angle of Attack in a Mach-6 Quiet Tunnel," AIAA Paper 2010-1062, 2010.

10. Kimmel, R., "Laminar-Turbulent Transition in a Mach 8 Elliptic Cone Flow," AIAA J., Vol. 37, No. 9, pp. 1080-87, 1999.

11. Poggie, J. and Kimmel, R., "Traveling Instability Waves in a Mach 8 Flow over an Elliptic Cone," AIAA J., Vol. 38, No. 2, pp. 251-58, 2000.

12. Kimmel, R. L., and Poggie, J., "Transition on an Elliptic Cone at Mach 8," American Society of Mechanical Engineers ASME FEDSM97-3111, June 1997.

13. Kimmel, R. L. and Poggie, J., "Three-Dimensional Hypersonic Boundary Layer Stability and Transition," Air Force Research Laboratory Technical Report, WL-TR-97-3111, Wright-Patterson Air Force Base, Ohio, December 1997.

14. Kimmel, R. L., Poggie, J. and Schwoerke, S. N., "Laminar-Turbulent Transition in a Mach 8 Elliptic Cone Flow," AIAA Journal, vol. 37, no. 9, pp. 1080-1087, Sep. 1999.

15. Schmisseur, J. D., "Receptivity of the Boundary Layer on a Mach-4 Elliptic Cone to Laser-Generated Localized Freestream Perturbations," Doctoral Dissertation, Purdue University Aerospace Sciences Laboratory, December 1997.

16. Holden, M., "Experimental Studies of Laminar, Transitional, and Turbulent Hypersonic Flows Over Elliptic Cones at Angle of Attack," Air Force Office of Scientific Research Technical Report AFRL-SR-BL-TR-98-0142, Bolling Air Force Base, DC, 1998.

17. Schmisseur, J. D., Schneider, S. P., and Collicott, S. H., "Response of the Mach 4 Boundary Layer on an Elliptic Cone to Laser-Generated Freestream Perturbations," AIAA Paper 1999-0410, January 1999.

18. Berger, K., Rufer, S., Kimmel, R., and Adamczak, D., "Aerothermodynamic Characteristics of Boundary Layer Transition and Trip Effectiveness of the HIFiRE Flight 5 Vehicle," AIAA Paper 2009-4055, 2009.

19. Choudhari, M., Chang, C.-L., Li, F., Berger, K., Candler, G., and Kimmel, R., "Transition Analysis for the HIFiRE-5 Vehicle," AIAA Paper 2009-4056, 2009.

20. Choudhari, M., Chang, C.-L., Li, F., Edwards, J., and Candler, G., "Hypersonic Boundary Layer Transition: Roughness Effects and 3D Configurations," NASA Fundamental Aeronautics Program Annual Meeting, Atlanta, GA, Oct. 5-7, 2008.

21. Juliano, T., Swanson, E. and Schneider, S.P., "Transition Research and Improved Performance in the Boeing/AFOSR Mach-6 Quiet Tunnel,” AIAA Paper 2007-0535, 2007.

22. Saric, W.S., Private Communication, 2009.

23. Wheaton, B. M., Juliano, T. J., Berridge, D. C., Chou, A., Gilbert, P. L., Casper, K. M., Sheen, L. E. and Schneider, S. P., "Instability and Transition Measurements in the Mach-6 Quiet Tunnel," AIAA Paper 2009-3559, 2009.

24. Li, F., Choudhari, M. M., Chang, C.-L., Wu, M. and Greene, P. T. "Development and Breakdown of Görtler Vortices in High Speed Boundary Layers," AIAA Paper 2010-705, 2010.

25. Balakumar, P., "Receptivity of Hypersonic Boundary Layers over Straight and Flared Cones," AIAA Paper 20101065, 2010.

26. http://vulcan-cfd.larc.nasa.gov/index.html (November 23, 2009)

27. van Albada, G. D., van Leer, B. and Roberts, W. W. , "A Comparative Study of Computational Methods in Cosmic Gas Dynamics," Astronomy and Astrophysics, Vol. 108, p76, 1982.

28. Edwards, J.R., “A Low-Diffusion Flux-Splitting Scheme for Navier-Stokes Calculations,” Computer and Fluids, Vol. 6, pp. 635-659, 1997.

29. Litton, D., Edwards, J., and White, J., “Algorithmic Enhancements to the VULCAN Navier-Stokes Solver," AIAA Paper 2003-3979, 2003.

30. Cheatwood, F.M. and Gnoffo, P.A., "User's Manual for the Langley Aerothermodynamic Upwind Relaxation Algorithm (LAURA)," NASA TM 4674, April 1996.

31. Chang, C.-L., "LASTRAC.3d: Transition Prediction in 3D Boundary Layers," AIAA Paper 2004-2542, 2004.

32. Li, F. and Choudhari, M., "Spatially Developing Secondary Instabilities and Attachment Line Instability in Supersonic Boundary Layers," AIAA Paper 2008-590, 2008.

33. Ng., L. and Erlebacher, G., "Secondary Instabilities in Compressible Boundary Layers," Phys. Fluids A, Vol. 4 (4), p. 710-26, April 1992.

34. Herbert, T., "Secondary Instability of Boundary Layers," J. Fluid Mech,. Vol. 20, p. 487, 1988.

35. Li, F., Choudhari, M., Chang, C.-L., Streett, C. L. and Carpenter, M. H., "Roughness Based Crossflow Transition Control: A Computational Assessment," AIAA Paper 2009-4105, 2009. 


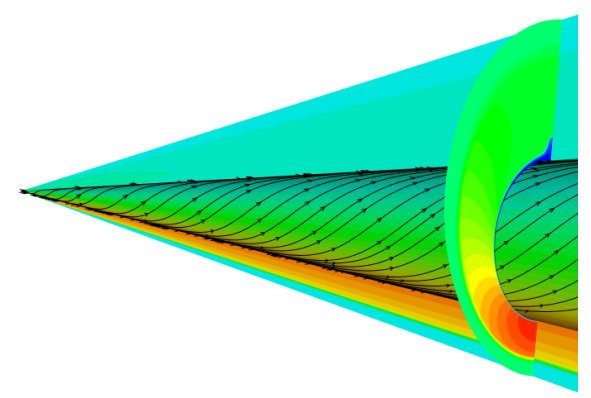

(a) Flow schematic depicting limiting streamlines (also referred to as skin friction lines), along with axial momentum contours along the symmetry plane and a cross sectional cut at $x=0.457 \mathrm{~m}$

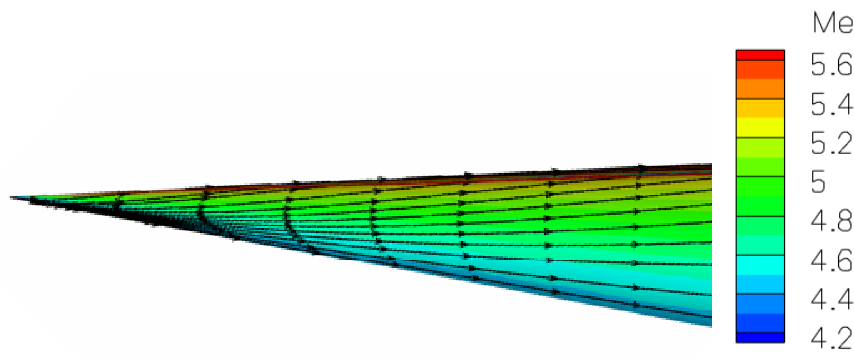

(b) Surface streamlines overlaid on flood contours of Mach number near boundary layer edge

Figure 1. Base flow for the Purdue cone configuration at 6 degree angle of attack
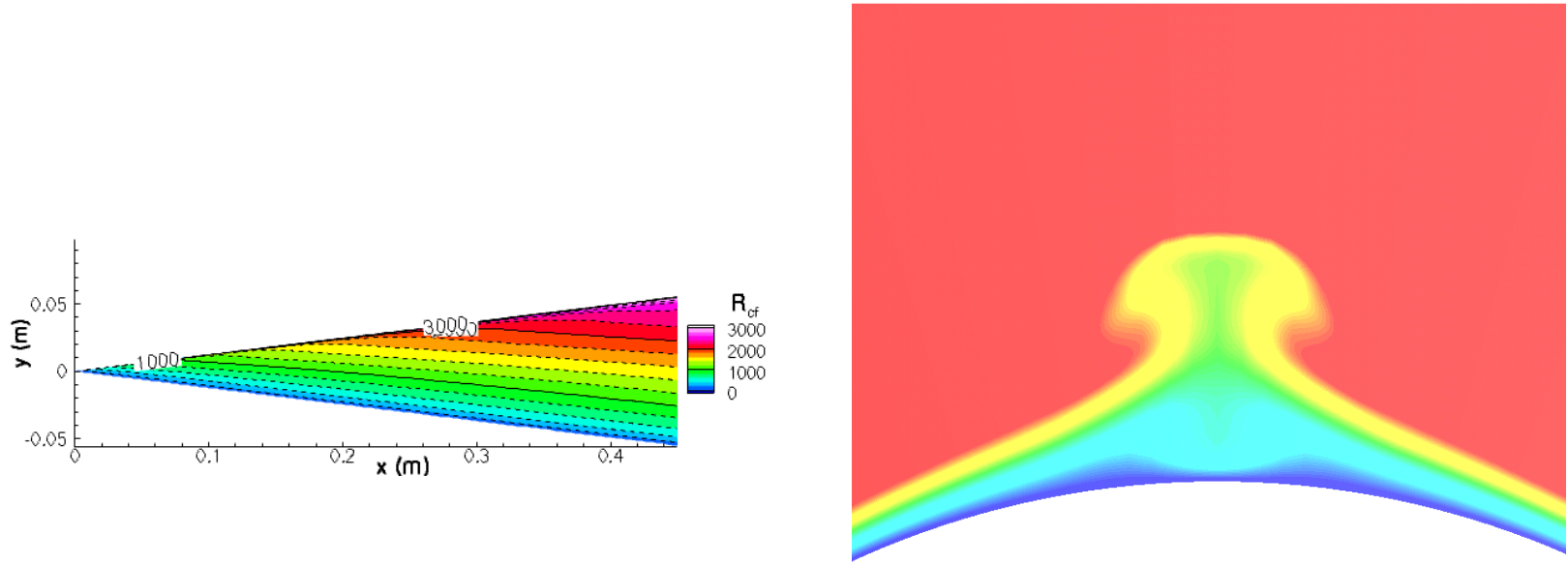

Figure 2. Crossflow Reynolds number distribution over the cone surface for the 6 degree angle of attack case

Figure 3. Representative Mach number contours in the vicinity of the leeward plane for the 6 degree angle of attack case 


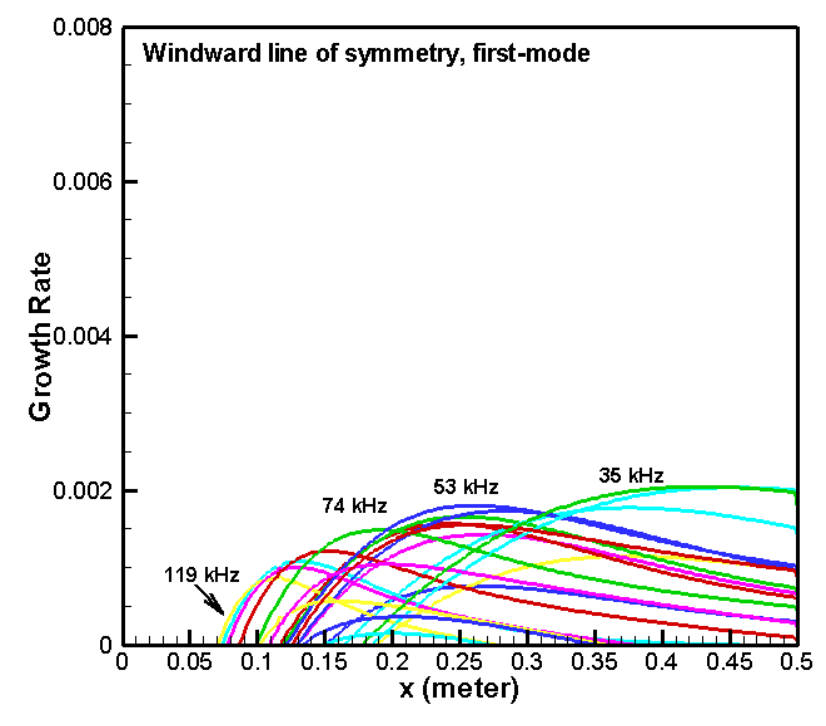

(a) Oblique first mode waves

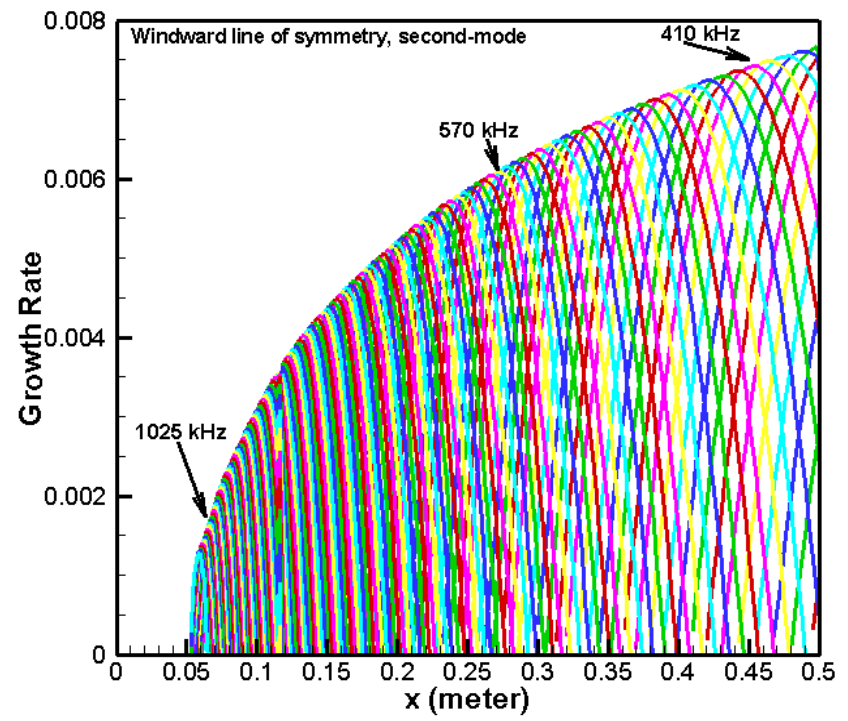

(b) 2D second mode waves

Figure 4. Growth rates of oblique first mode and 2D second mode instability waves along windward meridian for the 6 degree angle of attack case.

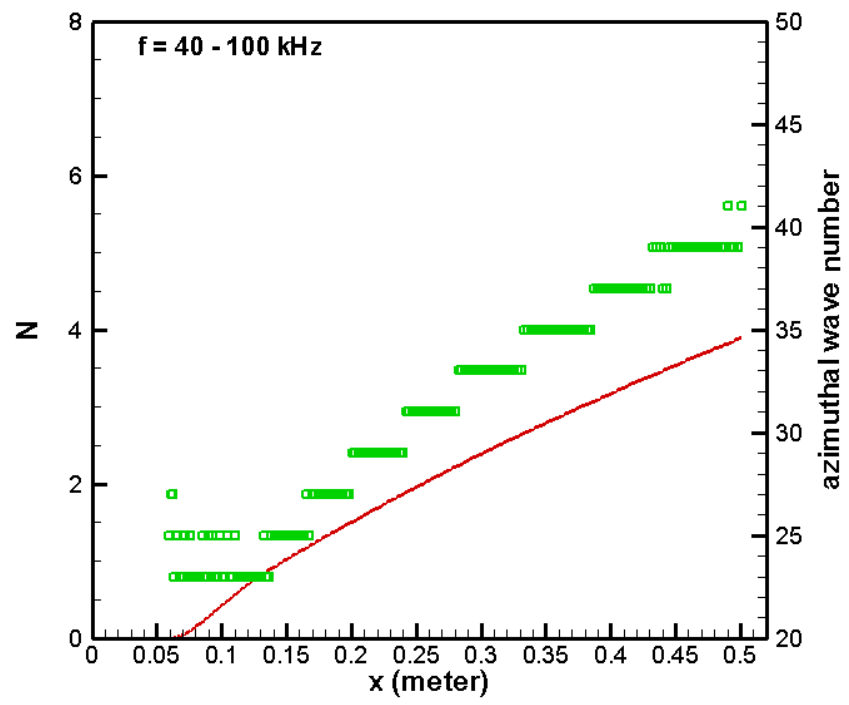

(a) Oblique first mode with constant azimuthal wave numbers.

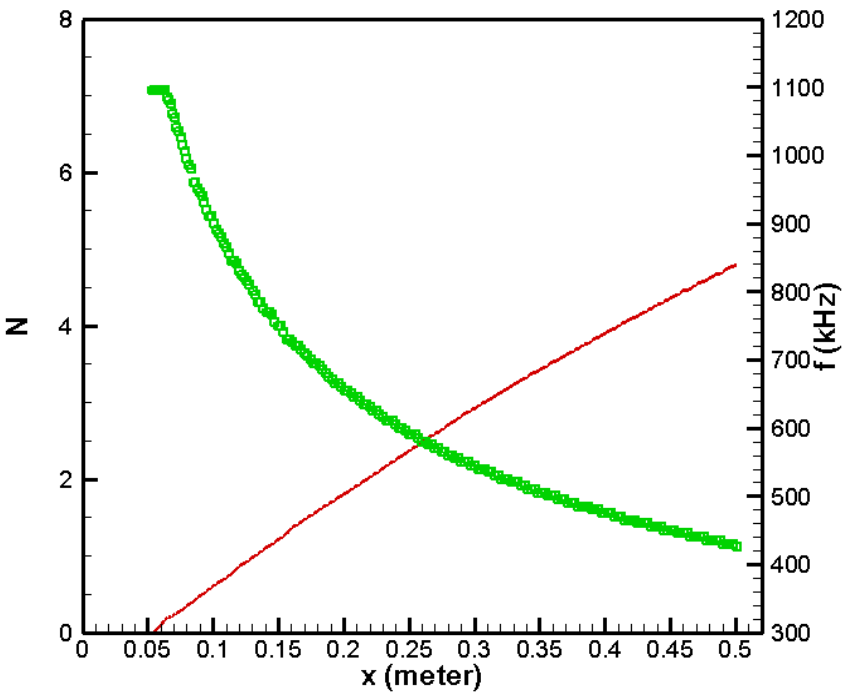

(b) 2D second mode.

Figure 5. Computed $\mathrm{N}$-factor envelop for disturbances with various frequencies and azimuthal wave numbers for the 6 degree angle of attack case. Red lines represent $\mathrm{N}$-factors, green symbols represent wavenumber or frequency. 


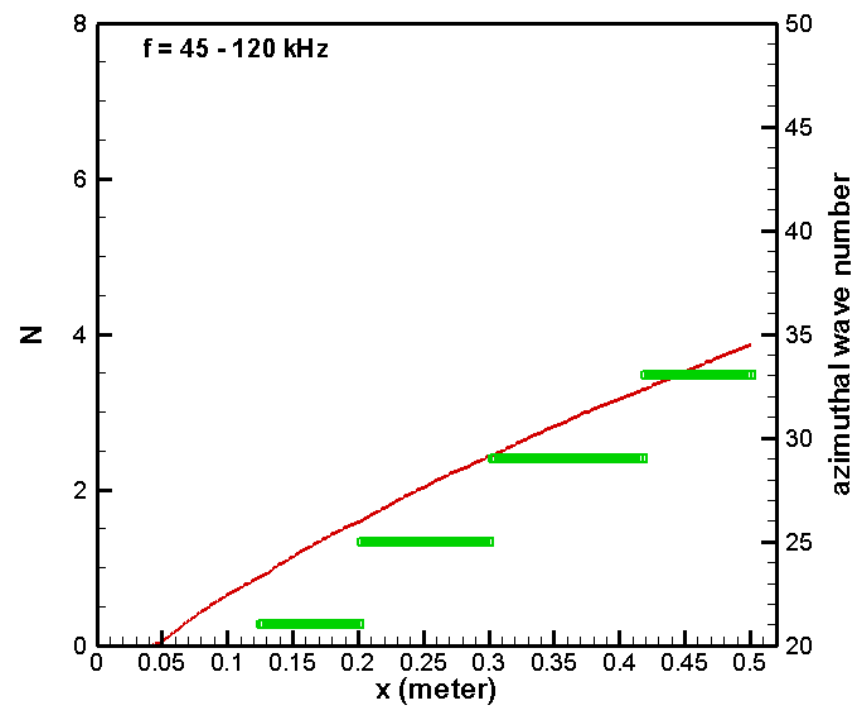

(a) Oblique first mode with constant azimuthal wave numbers

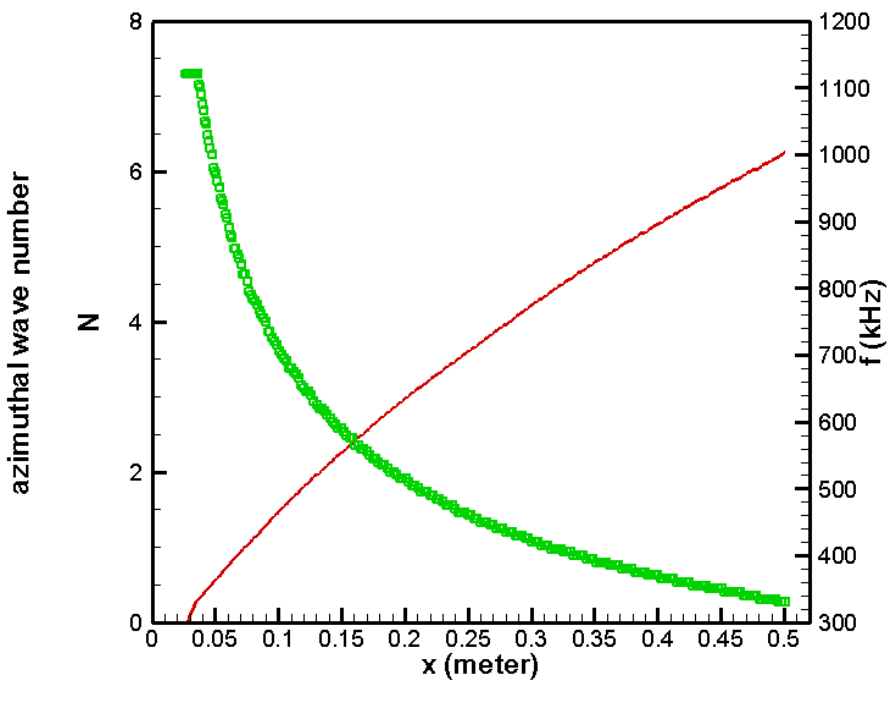

(b) 2D second mode

Figure 6. Computed $\mathrm{N}$-factor envelop for disturbances with various frequencies and azimuthal wave numbers for the 3 degree angle of attack case Red lines represent $\mathrm{N}$-factors, green symbols represent wavenumber or frequency.

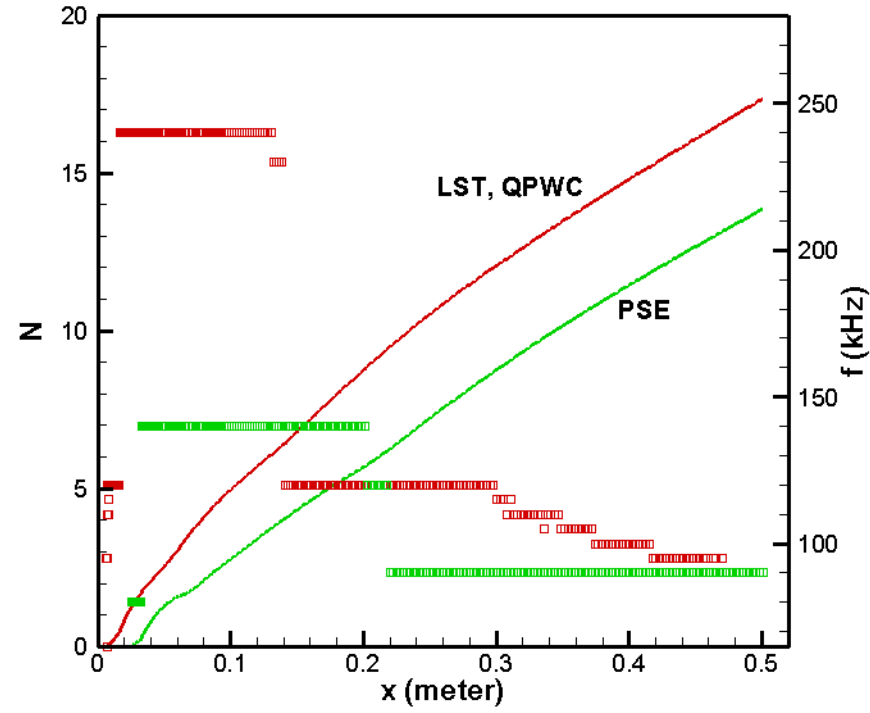

(a) 6 degree angle of attack

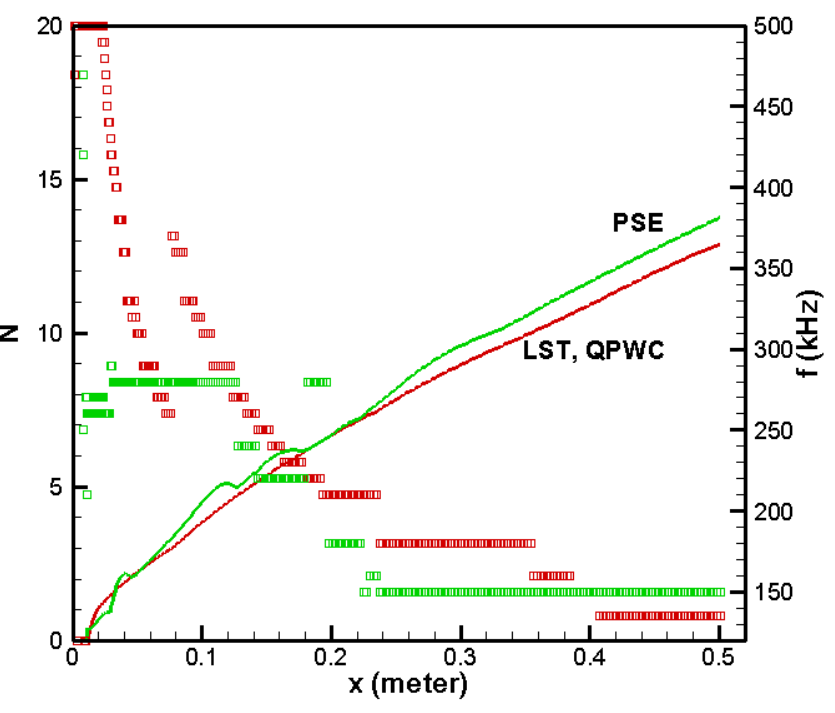

(b) 3 degree angle of attack

Figure 7. Computed N-factor envelope for disturbances with various frequencies using LST with curvature included and linear PSE: Red color represents results of quasi-parallel with curvature (QPWC) computations, and green color represents PSE results. Lines and symbols indicate $\mathrm{N}$-factors and frequency, respectively. 


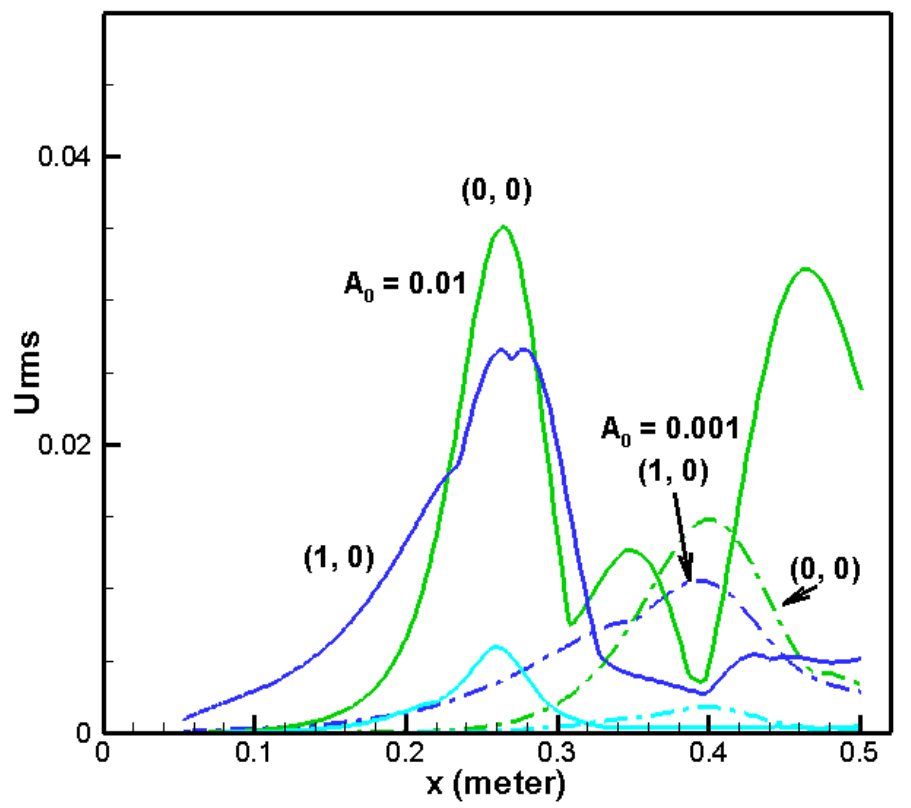

Figure 8. Nonlinear evolution of streamwise velocity perturbations for a $2 \mathrm{D}$ second mode disturbance with a frequency of $100 \mathrm{kHz}$ and two different initial amplitudes. Here, $(1,0)$ and $(0,0)$ refer to the fundamental mode of the disturbance and the mean flow correction, respectively.

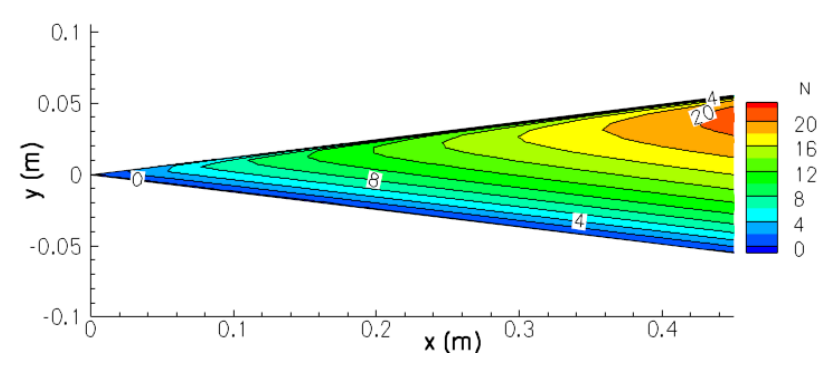

(a) $\mathrm{N}$-factor contours over cone surface

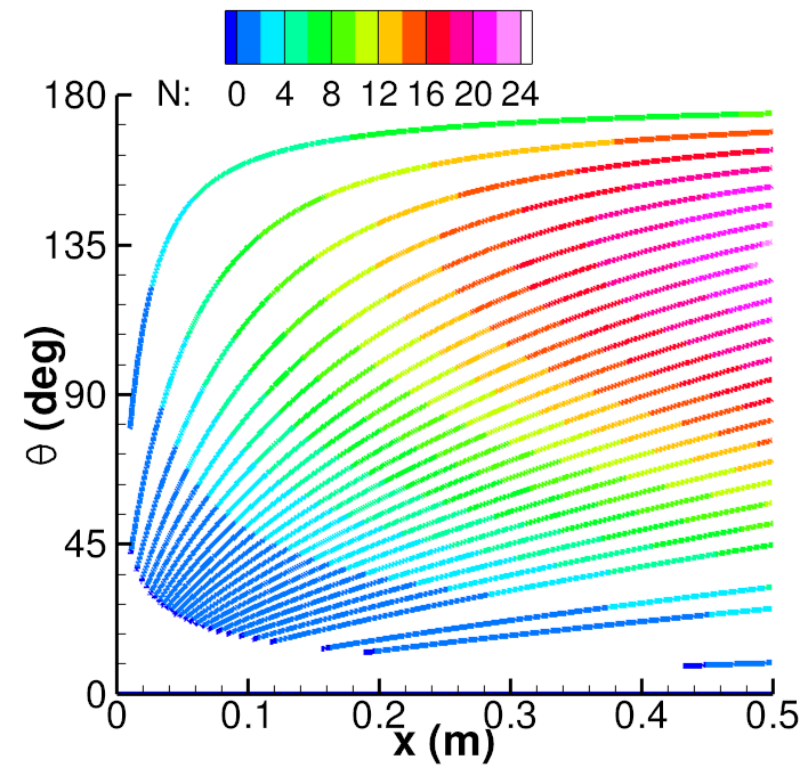

(b) $\mathrm{N}$-factor contours in $\mathrm{x}-\theta$ plane

Figure 9. Amplification factors for stationary crossflow vortex instabilities 


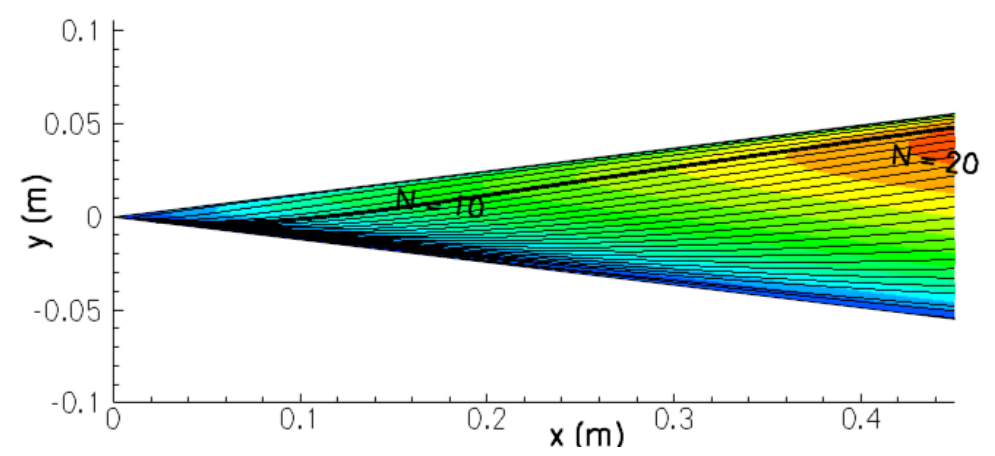

(a) $\mathrm{N}$-factor contours with integration trajectories. The thicker black line indicates the particular trajectory along which computational results shown in (b) are obtained.

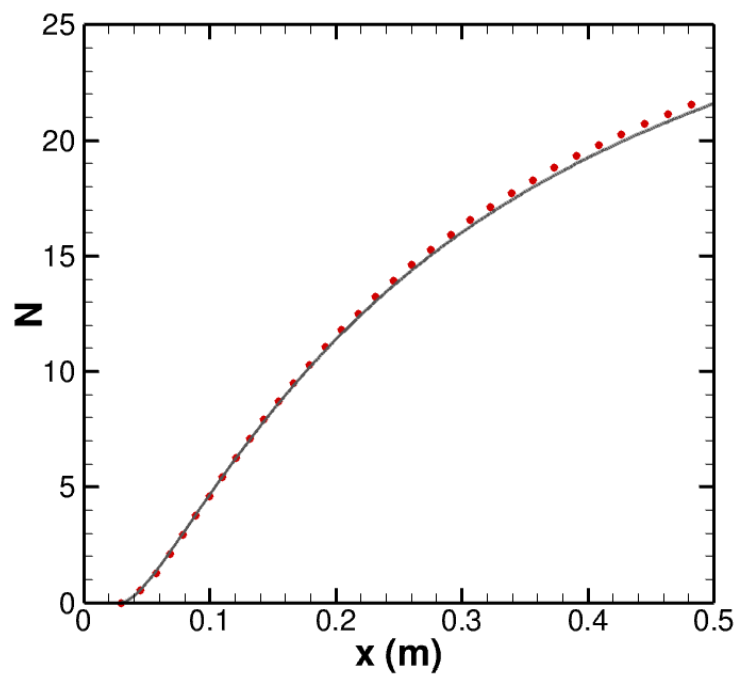

(b) Effect of non-parallelism on $\mathrm{N}$-factor curves for stationary crossflow instability along a selected disturbance trajectory from (a); the azimuthal wave number corresponds to $\beta=42$. The line curve denotes the predicted disturbance growth without non-parallel effects; symbols denote results with the non-parallel effects included.

Figure 10. Amplification factors for stationary crossflow vortex instabilities

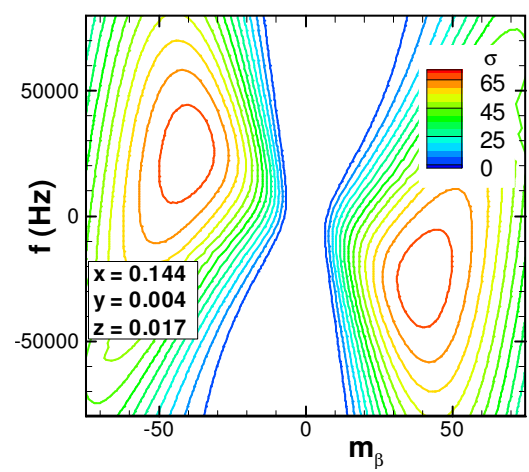

(a) $x \approx 0.15 \mathrm{~m}$

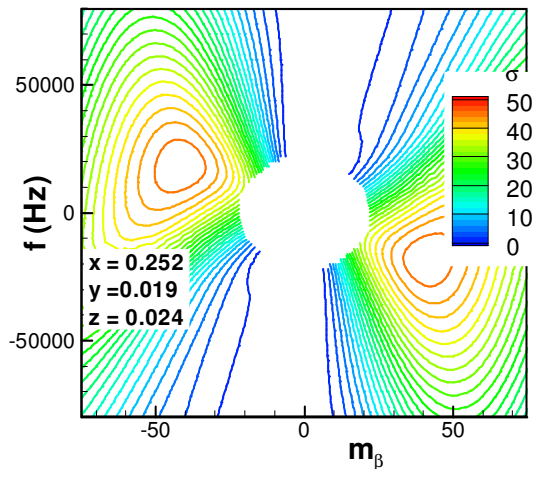

(b) $x \approx 0.25 \mathrm{~m}$

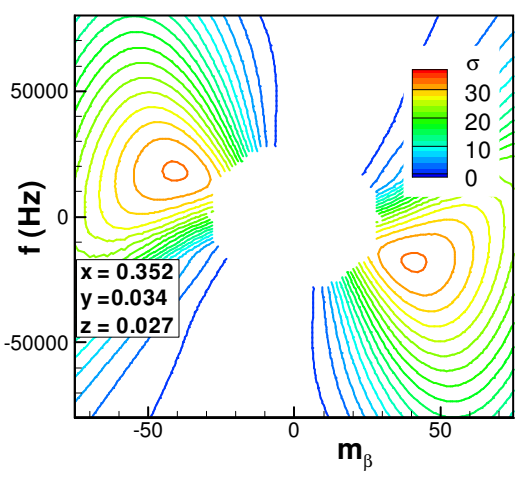

(c) $\mathrm{x}=0.35 \mathrm{~m}$

Figure 11. Growth rate variation of traveling crossflow vortex instabilities at selected locations along the disturbance trajectory highlighted by the thick black line in Fig. 10 (a). 


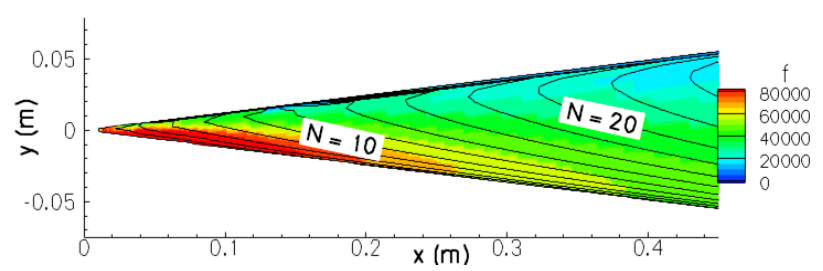

a) Flood contours of frequency of most amplified disturbance, superposed with line contours of $\mathrm{N}$-factor

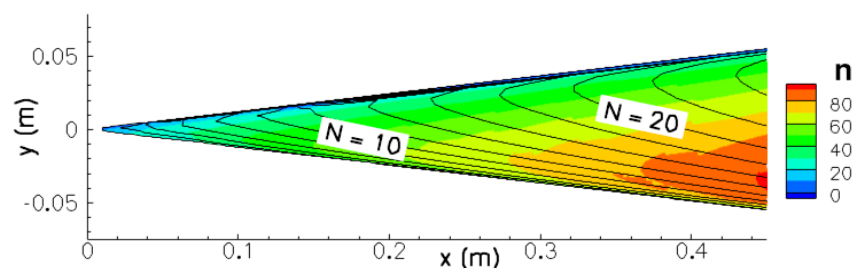

(b) Flood contours of most amplified azimuthal wavenunber $\mathrm{n}$, superposed with line contours of $\mathrm{N}$-factor

Figure 12. Amplification factors for traveling crossflow vortex instabilities

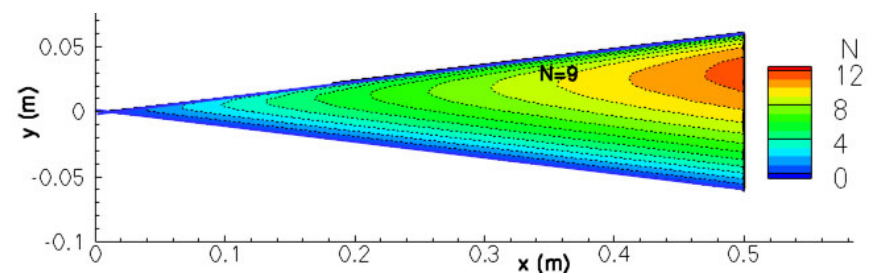

(a) Contours of $\mathrm{N}$-factor

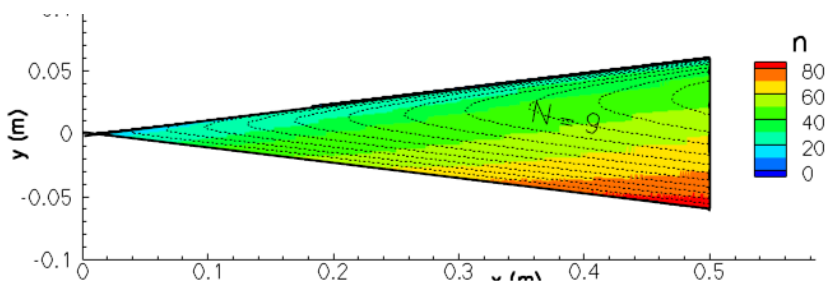

(b) Flood contours of most amplified azimuthal wave number $\mathrm{n}$, superposed with line contours of $\mathrm{N}$-factor

Figure 13. Stationary crossflow vortex instabilities at 3 degree angle of attack.

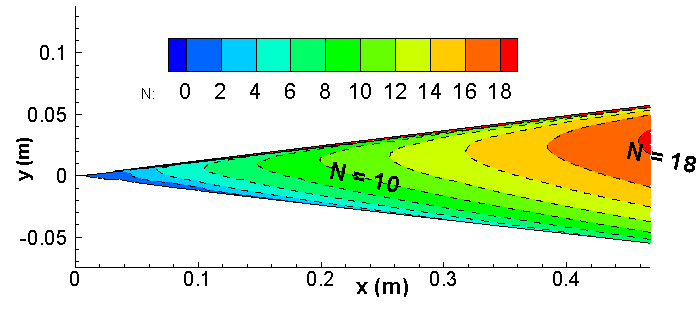

(a) Contours of $\mathrm{N}$-factor

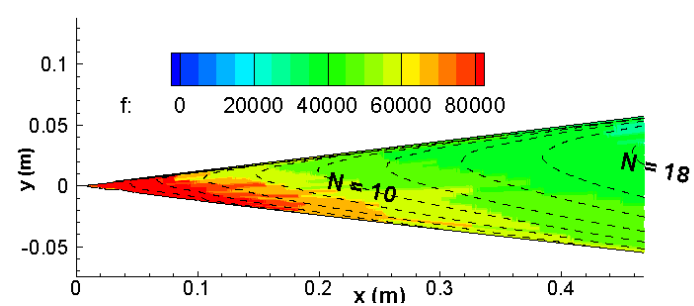

(c) disturbance frequency

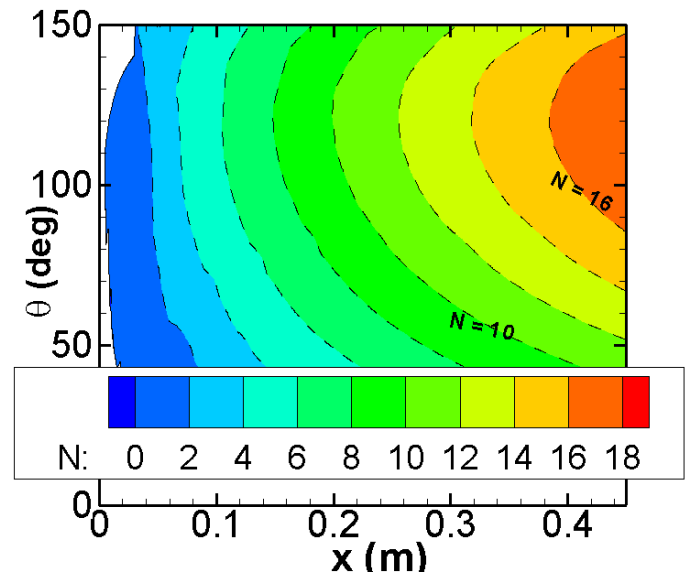

(b) $\mathrm{N}$-factor contours in $\mathrm{x}-\theta$ plane

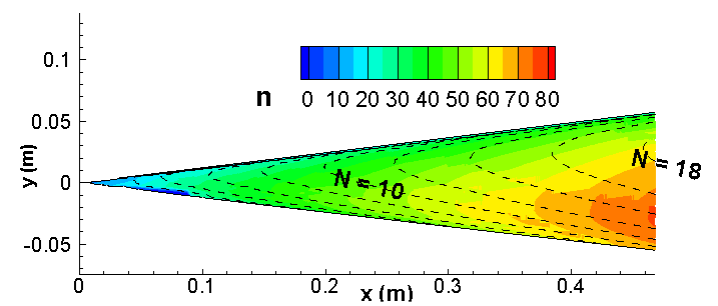

(d) azimuthal wave number

Figure 14. Traveling crossflow vortex instabilities at 3 degree angle of attack 


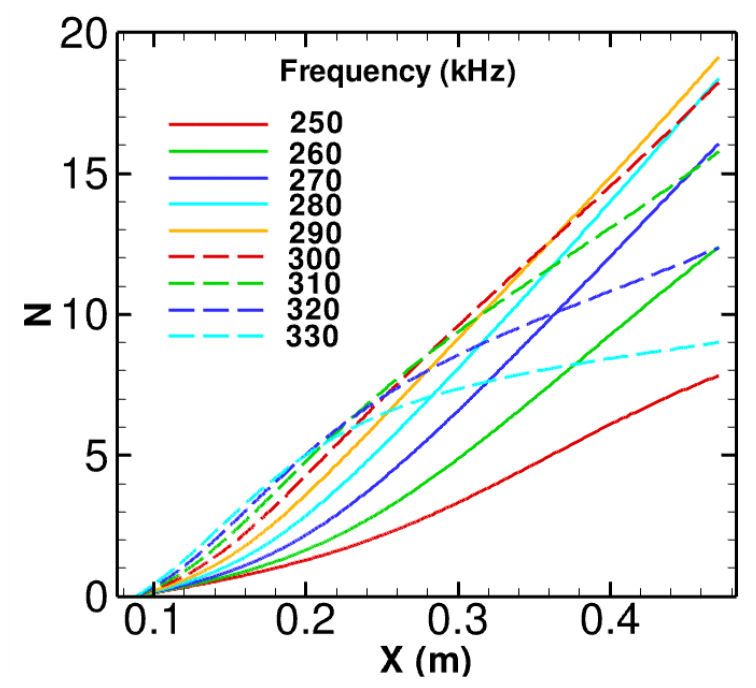

(a) $\mathrm{N}$-factors of second modes at various frequencies.

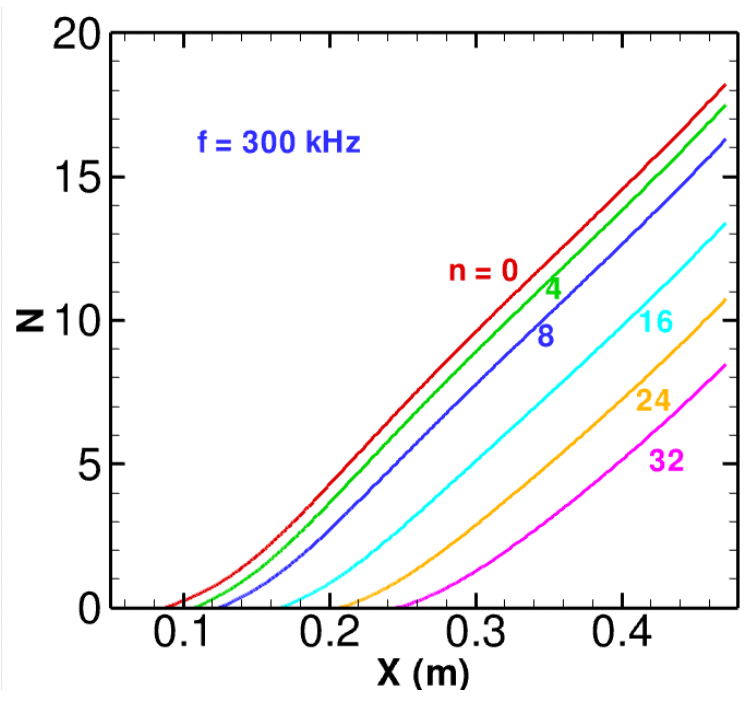

(b) $\mathrm{N}$-factors for oblique second modes of $300 \mathrm{kHz}$.

Figure 15. Linear N-factors of second mode for the Mach 6 compression cone.

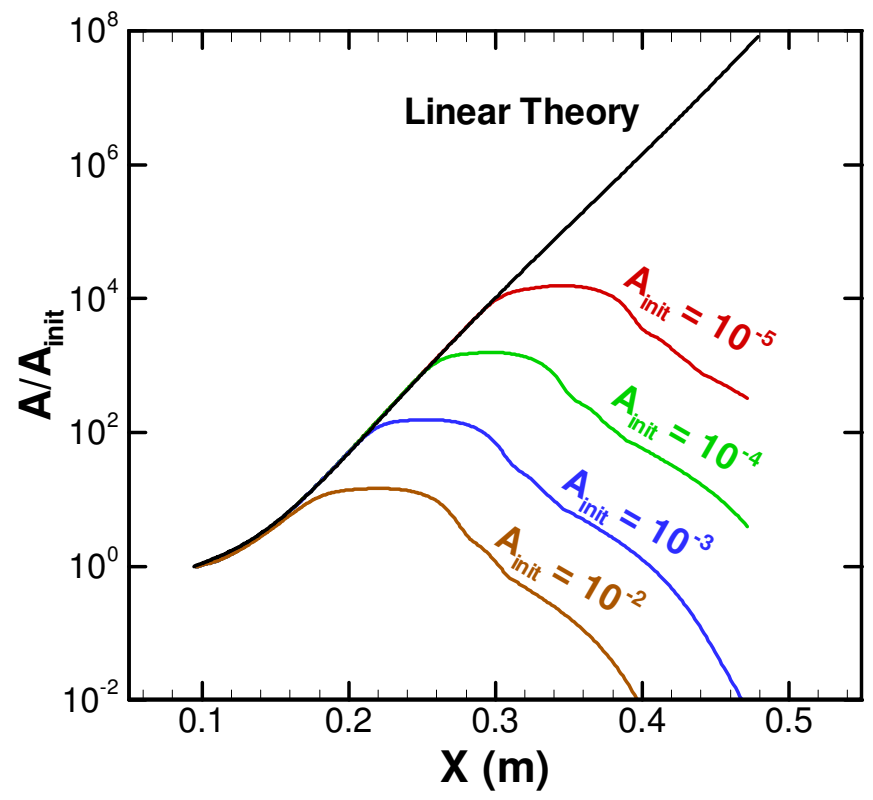

Figure 16. Normalized amplitude showing deviations from linear theory, and rapid decay in the downstream region. 


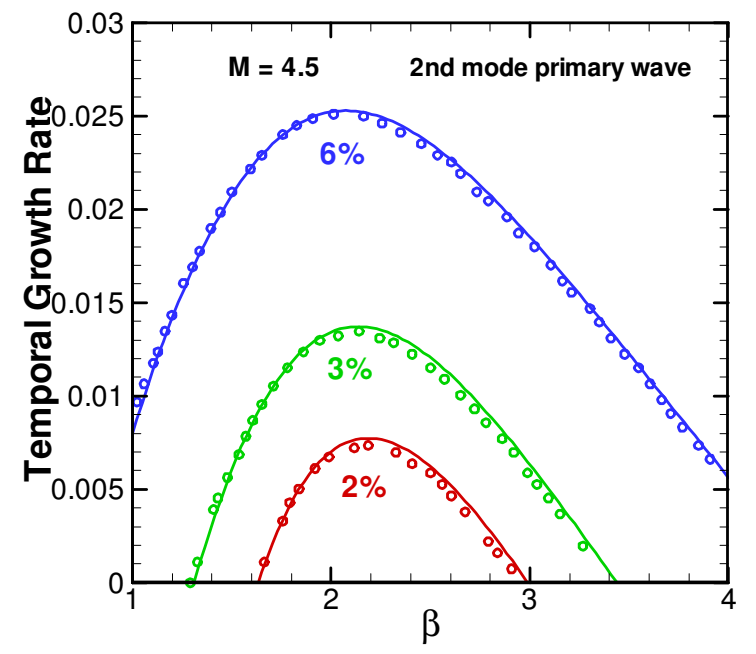

(a) Subharmonic instabilities based on a 2D second mode primary wave. The red, green, and blue curves denote increasing local amplitudes of the primary wave $(2 \%, 3 \%$, and $6 \%$, respectively).

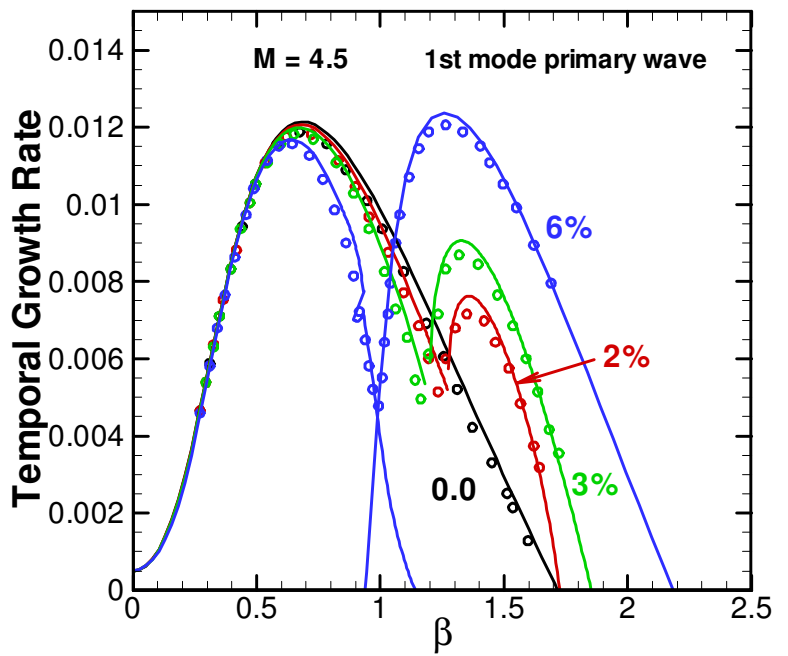

(b) Subharmonic instabilities based on 2D first mode wave.

Figure 17. Comparison of secondary instability growth rates for a Mach 4.5 flat plate boundary layer with Ref. 33. The temporal growth rate is plotted against spanwise wavenumber $\beta$. Lines denote present results, whereas symbols indicate results from Fig. 12 in Ref. 33. Details of flow conditions and normalization of temporal growth rate and wavenumber $\beta$ are given in Ref. 33 .

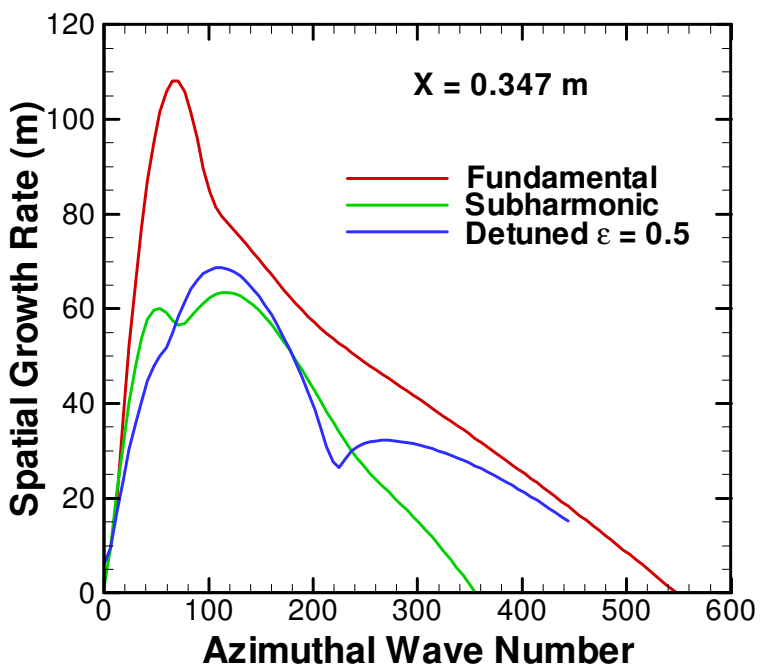

Figure 18. Growth rates of secondary instability modes at a fixed streamwise location. Initial amplitude of the primary second mode wave, measured in terms of peak axial velocity fluctuation relative to the freestream velocity, is equal to $10^{-5}$. 


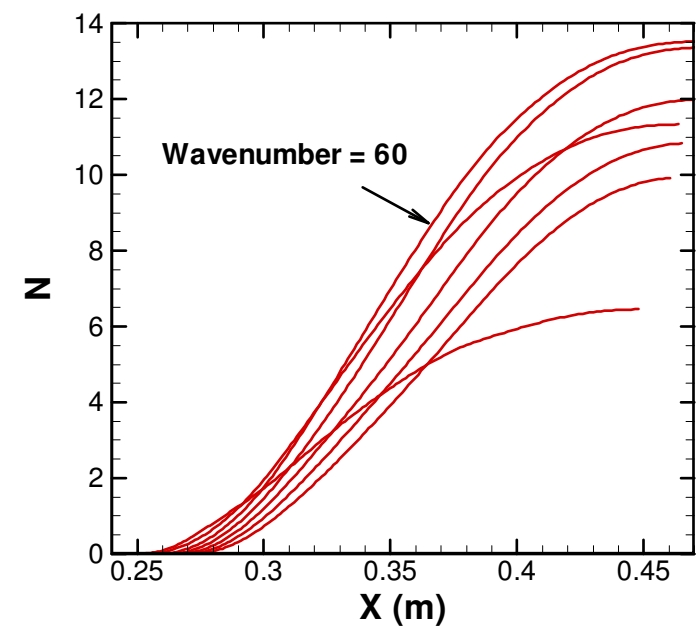

(a) Fundamental secondary instability.

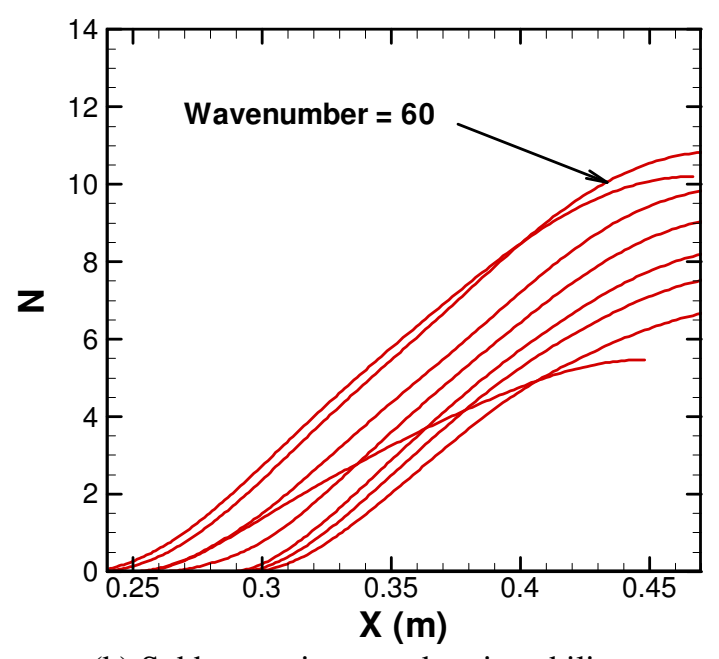

(b) Subharmonic secondary instability.

Figure 19. Linear $\mathrm{N}$-factors of secondary instability riding on a second mode of $300 \mathrm{kHz}$ in frequency.

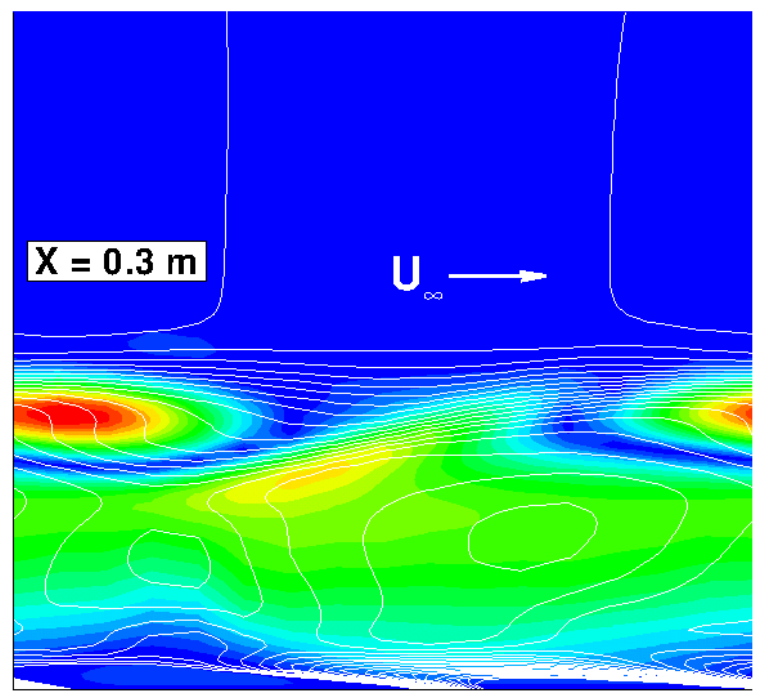

(a) Fundamental

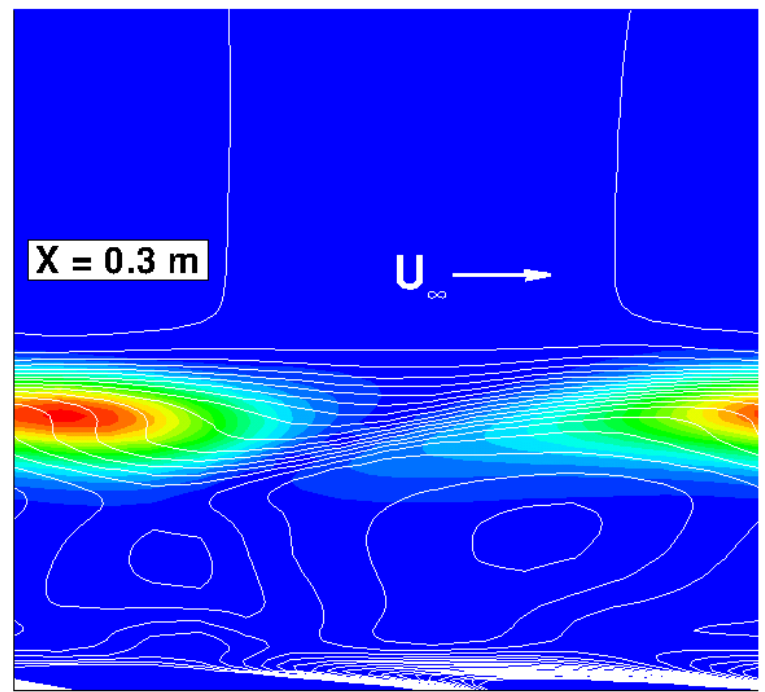

(b) Subharmoinic

Figure 20. Eigenfunction shapes of streamwise velocity fluctuations. The abscissa corresponds to one spanwise wavelength of the secondary instability mode, whereas the ordinate corresponds to the wall-normal direction. The background white lines represent contours of mean shear $\mathrm{dU} / \mathrm{dy}$. The base flow is from left to right. 


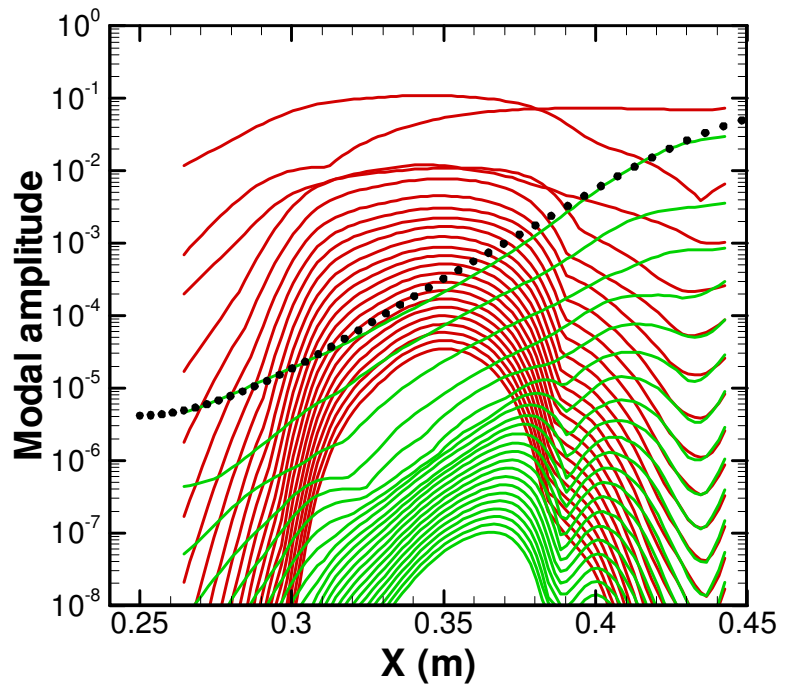

(a) Modal amplitudes of second mode (red), and secondary instability (green), computed via nonlinear PSE. The black dots represent linear theory prediction.

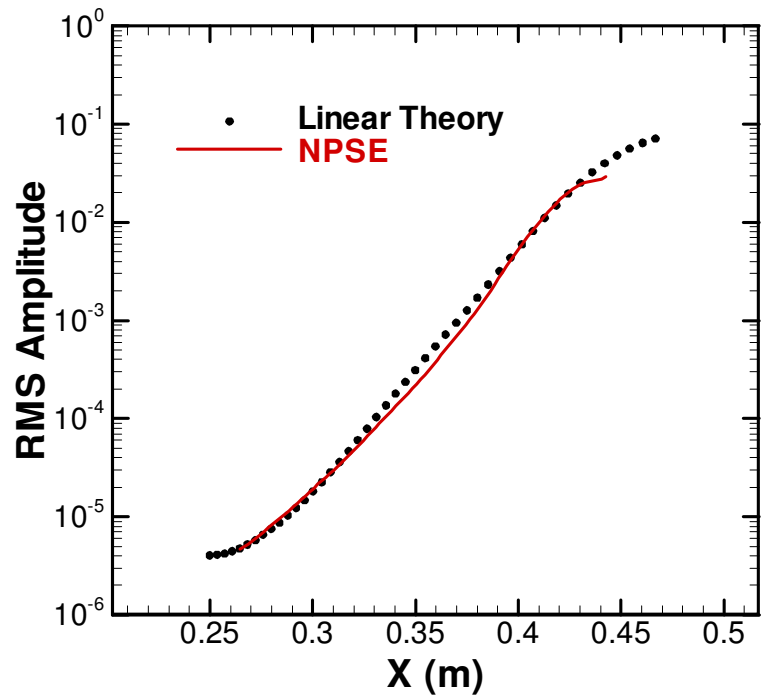

(b) RMS amplitude of secondary instability computed by nonlinear PSE and linear theory.

Figure 21. Amplitude evolution of second mode and subharmonic secondary instability.

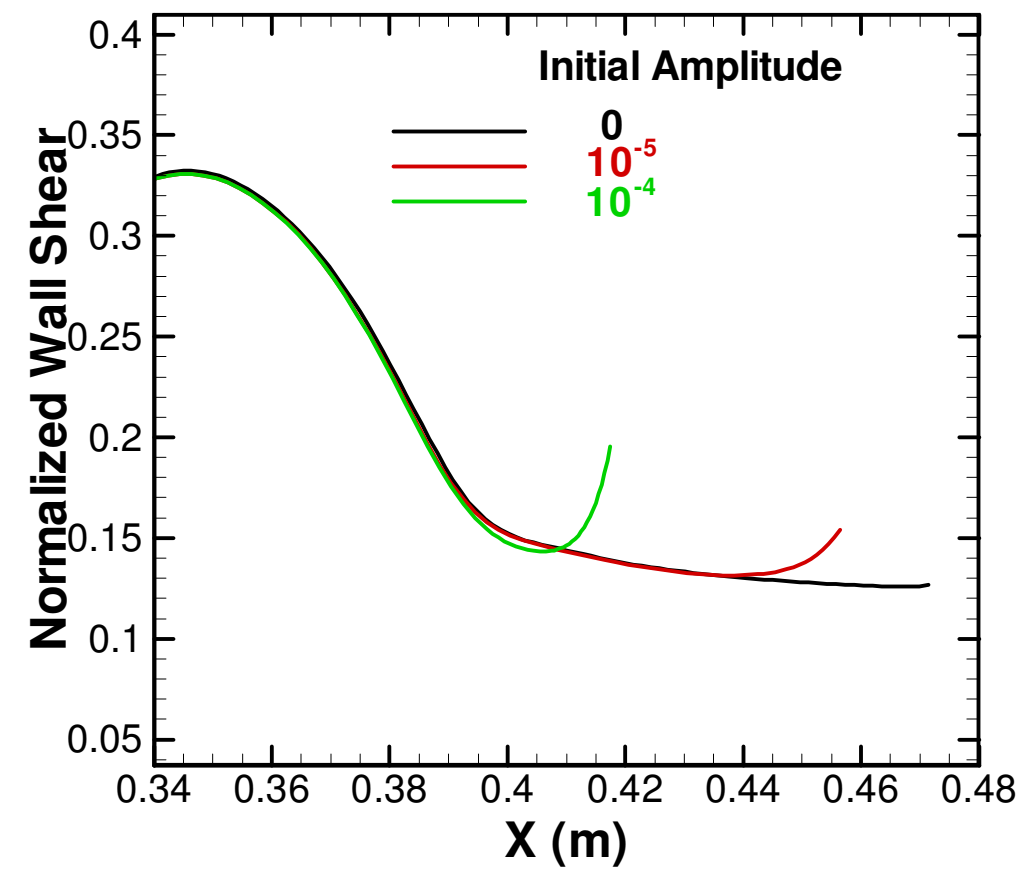

Figure 22. Evolution of normalized wall-shear for various initial amplitudes of secondary instability. 

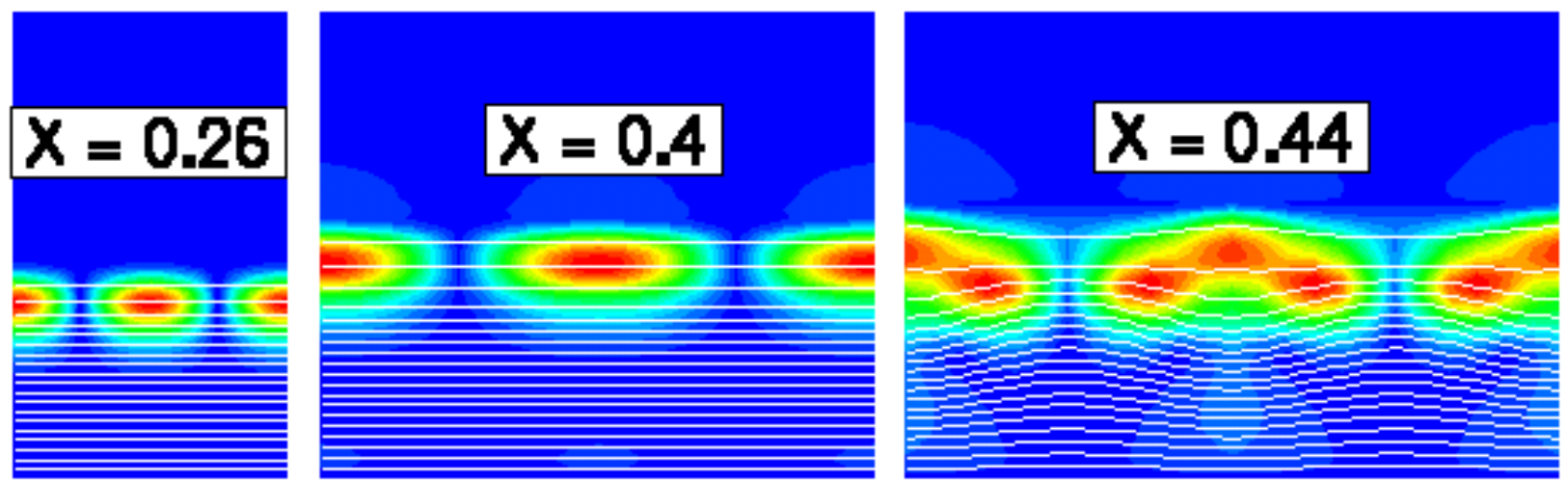

Figure 23. Contours of root mean square (RMS) streamwise velocity fluctuation at three streamwise locations. At each location, the fluctuation amplitude is normalized by the value of its local peak. Blue color denotes zero fluctuations, whereas the red color denotes the peak velocity fluctuations. The background white lines represent contours of streamise velocity (coming out of paper).

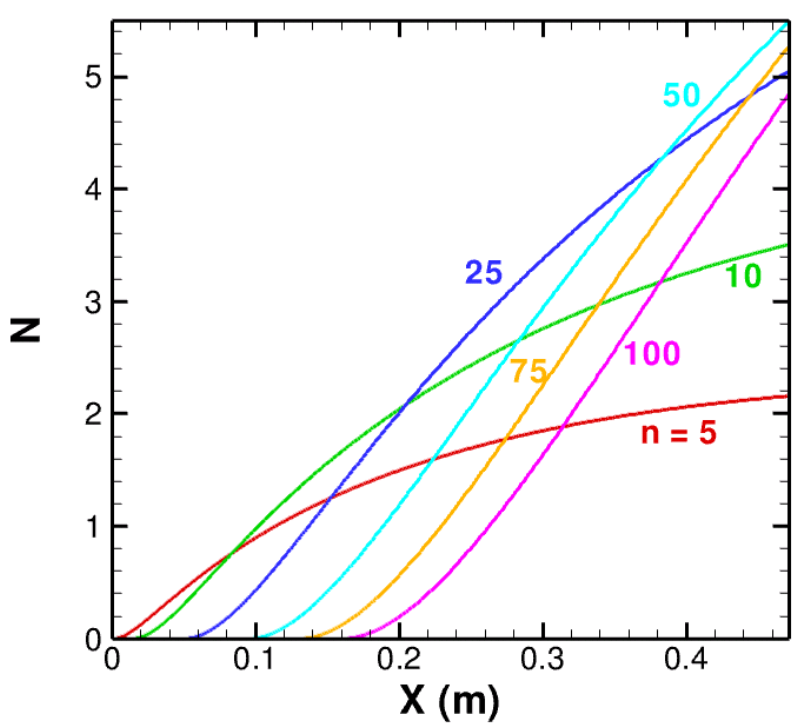

Figure 24. N-factors of linear Görtler instability at various azimuthal wavenumbers

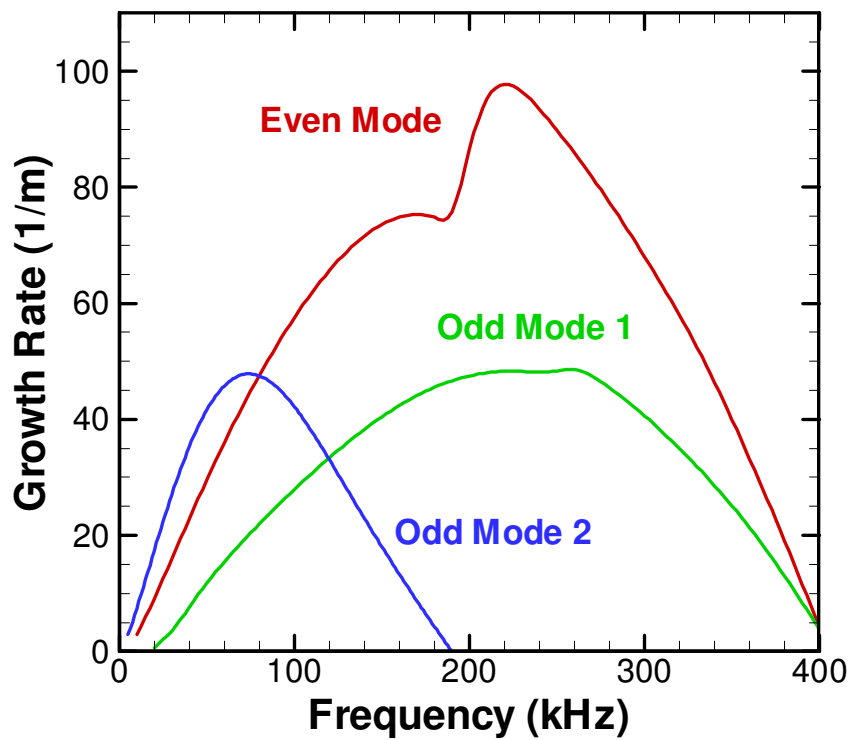

Figure 25. Growth rates of secondary instability at $\mathrm{x}=0.43$ $\mathrm{m}$. 


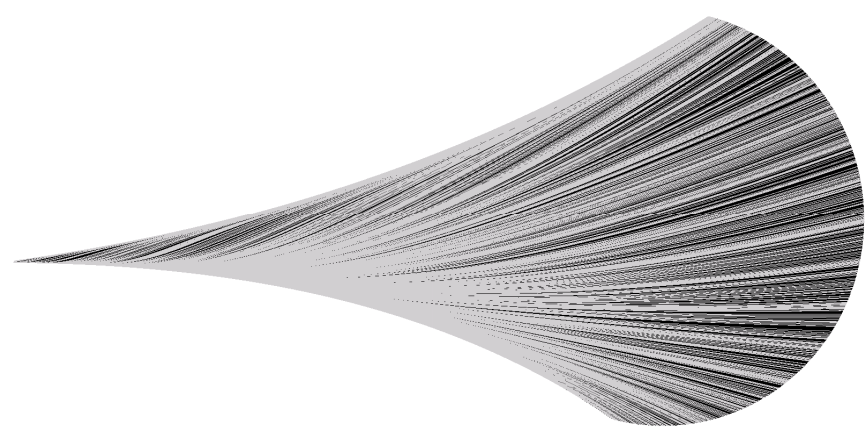

Figure 26. Surface friction lines at 0.5 degree angle of attack.

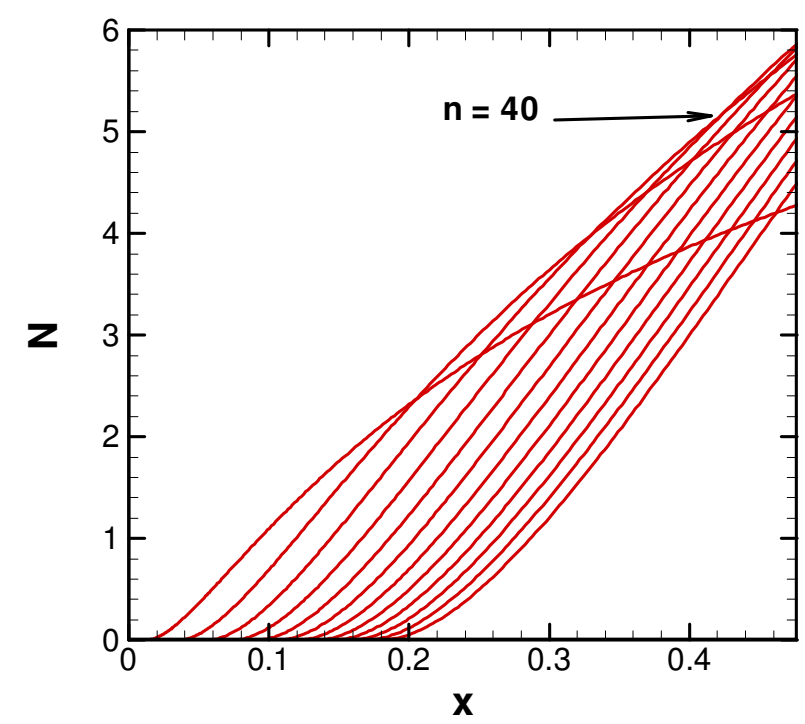

(a) Leeward

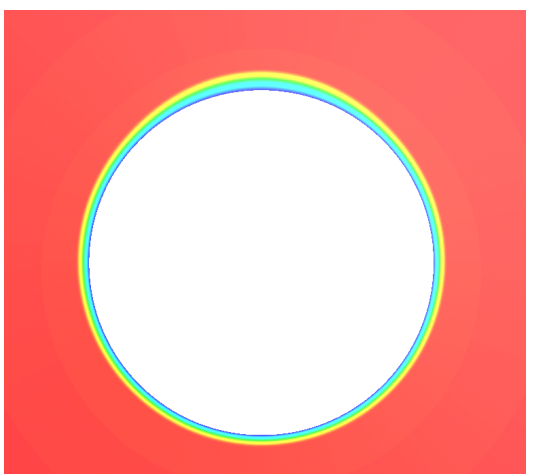

Figure 27. Streamwise velocity contours in a crossplane at $x=0.2 \mathrm{~m}$. Leeward side is on top, showing a thicker boundary layer than the windward side.

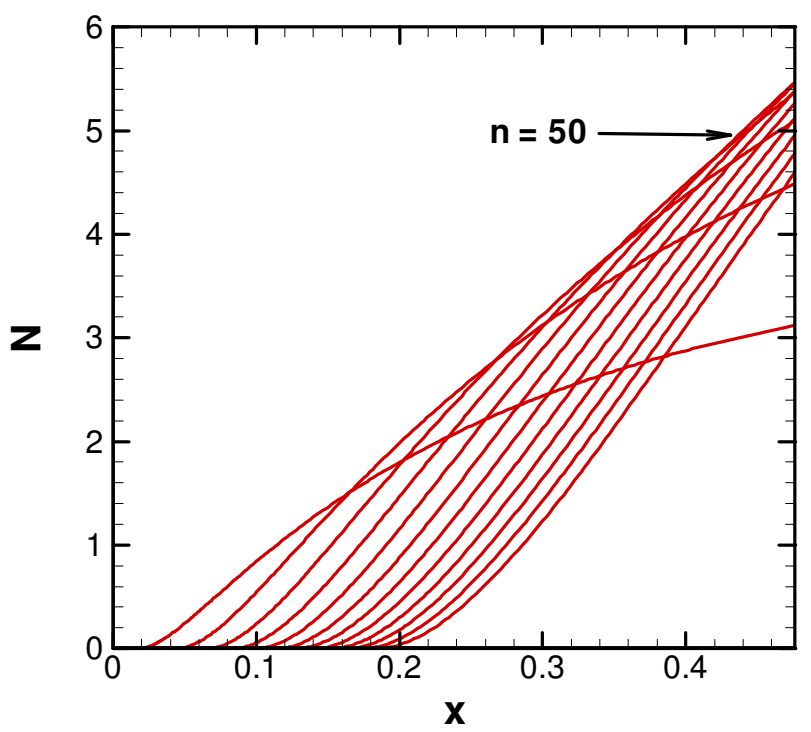

(b) Windward

Figure 28. Effect of small angle of attack (0.5 degrees) on $\mathrm{N}$-factor of linear Görtler instability modes. Each curve represents one azimuthal wavenumber, $n$, from $n=10$ to $n=120$ in increments of 10 . The leeward side is slightly more unstable. 


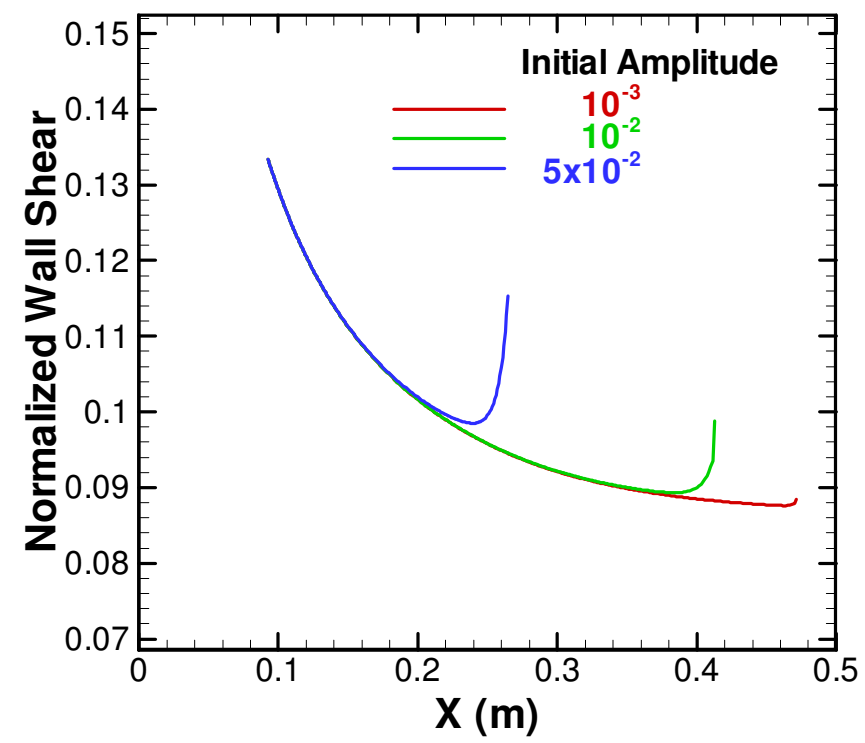

Figure 29. Wall shear stress in the case of oblique first mode interaction for selected values of initial amplitude. Initial amplitude is measured in terms of peak axial velocity perturbation at the neutral location, scaled by the freestream velocity. Relatively large initial amplitudes are necessary to initiate the onset of transition, which is indicated by the rapid rise in wall shear. 